\author{
Pedro Antonio Merino García* \\ Rebeca Albacete Sánchez-Mateos ${ }^{* *}$ \\ José María Martínez Pérez ${ }^{* *}$ \\ Juan Jesús Rubio Jareño**
}

\title{
RECUPERACIÓN ECONÓMICA Y SECTOR EXTERIOR ESPAÑOL, ALGUNAS CONSIDERACIONES
}

En este artículo se pasa revista a los cambios de los últimos 20 años del comercio internacional para, a continuación, analizar el comportamiento del sector exterior español antes, durante y después de la gran crisis financiera mundial (GFC, por sus siglas en inglés) que se inició en 2008. Posteriormente, se analiza si durante el periodo poscrisis, a partir de 2014, se ha producido un cambio en las elasticidades del sector exterior (tanto español como europeo) y si los cambios regulatorios y los comportamientos de los agentes económicos se han traducido en un comportamiento diferencial español frente a nuestros socios europeos, que nos ayude a realizar hipótesis de lo que pueda ocurrir con el sector exterior en la recuperación pospandemia COVID-19.

\section{Economic recovery and Spanish foreign sector, some considerations}

This article reviews the changes of the last 20 years in international trade, and then analyzes the behavior of the Spanish foreign sector before, during and after the great global financial crisis (GFC) that began in 2008. Subsequently, analyzes whether during the post-crisis period, starting in 2014, there has been a change in the elasticities of the foreign sector (both Spanish and European) and whether the regulatory changes and the behavior of economic agents have translated into a behavior Spanish differential compared to our European partners that helps us to hypothesize what may happen to the foreign sector in the post-COVID-19 pandemic recovery.

Palabras clave: sector exterior, comercio, valor añadido doméstico, valor añadido foráneo, España, crisis económica, pandemia, shocks, perspectivas macroeconómicas.

Keywords: external sector, trade, domestic added value, foreign added value, Spain, economic crisis, pandemic, shocks, macroeconomic prospects.

JEL: E66, F14, F40, F44.

\footnotetext{
* Director del Servicio de Estudios de Repsol.

** Economista Sénior del Servicio de Estudios de Repsol.

Versión de enero de 2022.

https://doi.org/10.32796/ice.2022.924.7350
} 


\section{Introducción}

En este trabajo se pasa revista a los cambios de los últimos 20 años del comercio internacional para, a continuación, analizar el comportamiento del sector exterior español antes, durante y después de la gran crisis financiera mundial (GFC, por sus siglas en inglés) que se inició en 2008. La crisis en el caso de España se tradujo en una fuerte caída del Producto Interior Bruto (PIB) — que no recuperó niveles precrisis hasta 2019y en un creciente peso del sector exterior en la economía, que contribuyó de manera muy positiva a la recuperación ante el colapso de la construcción y de las rentas domésticas.

Posteriormente, se analiza si durante el periodo poscrisis, a partir de 2014 , se ha producido un cambio en las elasticidades del sector exterior (tanto español como europeo) y si los cambios regulatorios y los comportamientos de los agentes económicos se han traducido en un comportamiento diferencial español frente a nuestros socios europeos, que nos ayude a realizar hipótesis de lo que pueda ocurrir con el sector exterior en la recuperación pospandemia COVID-19.

Para ello el documento se organiza, en primer lugar, en un apartado sobre las relaciones de crecimiento y comercio y la importancia de analizar el valor añadido y disminuir las discrepancias existentes en los datos de comercio internacional (apartado 2).

En el apartado 3, se comentará la contribución del sector exterior a la recuperación del PIB entre 2014-2019 y su composición regional. A continuación, en el apartado 4 se menciona y analiza qué cambios se producen con la crisis GFC en inflación, costes y crecimiento para los principales socios del euro. En concreto se analizan los cambios en inflación diferencial (devaluación competitiva; costes laborales y productividad), así como los cambios regulatorios que puedan explicar el importante papel del sector exterior en la recuperación, comentando si estos indicadores han mejorado o empeorado en los últimos años 2017-2019, y si pueden volver a mejorar tras la crisis del COVID-19, lo que significaría una mayor aportación del sector exterior al crecimiento español.

En el apartado 5, se realiza un análisis descriptivo, pero completo, de los cambios en las elasticidades de exportaciones y de importaciones en España antes y después de la GFC, y se compara con lo sucedido en los principales países del euro.

En el apartado 6, se analizan los datos de comercio exterior en valor añadido con la fuente OCDE TiVA para ver si la mejora en valor añadido es mayor que en datos brutos, y si se corroboran los datos de cambios en las elasticidades. Para ello se ha utilizado la última versión de la base de datos, actualizada el pasado noviembre, y que ahora incluye información hasta el periodo 2018-2019. Además, el estudio de los datos en valor añadido arrojará luz sobre algunas tendencias del sector exterior, por ejemplo, por el mayor papel de los servicios, o la mayor integración intraindustrial en Europa.

En los apartados 7 y 8 se analizan los efectos potenciales de la crisis COVID-19 sobre el comercio internacional y los efectos de la distinta naturaleza de la GFC de 2008, crisis de exceso de endeudamiento y de inversión con restricción posterior de demanda y de financiación, frente a la actual crisis COVID-19, con caídas del consumo, pero no de rentas y rápida recuperación. La comparativa por sectores en España y la zona euro también es clave para entender cómo será el comercio exterior. Como es lógico hay que intentar entender las implicaciones de la distinta velocidad de recuperación por regiones, en función de los avances en la reducción de las patologías COVID-19 en el mundo y de las distintas políticas económicas monetarias y financieras. Hay un componente diferencial por todos conocidos en la crisis COVID-19: en España las exportaciones de servicios turísticos son claves tanto en la crisis como en la recuperación. Pero supondremos que se recupera en 2022.

Finalmente, se indican unas breves conclusiones en el apartado 9. En cualquier caso, muchas de las conclusiones de este análisis son tentativas, y casi 
hipótesis a contrastar con más trabajos, sobre todo dado el nivel de incertidumbre existente sobre el ritmo de recuperación y sobre los cambios potenciales en las cadenas de valor o en la recuperación de las tendencias existentes en el periodo 2015-2019.

\section{Cambios en la relación entre crecimiento y comercio tras la crisis financiera global}

Aunque en una tendencia ascendente desde la década de 1970, la globalización cobró velocidad en la década de 1990, entrando en un periodo de «hiperglobalización» (Rodrik, 2011).

El auge de la globalización ha hecho aún más patente que nunca la relación de interdependencia entre el comercio internacional y el crecimiento económico. El comercio afecta al crecimiento a través de diferentes canales. Por un lado, de forma directa a través de la contribución directa de las exportaciones netas en el PIB; pero, además, hay que considerar que hay efectos de arrastre hacia la actividad interna, y que la configuración sectorial domestica viene determinada en cierta medida por necesidades de especialización ante la competencia internacional. Por otro lado, el comercio internacional y la liberalización económica son también un motor de la productividad total de los factores y, por tanto, del crecimiento potencial. En este sentido, un aumento del comercio suele asociarse con: una aceleración de la transferencia tecnológica; mejora en la eficiencia de la asignación de recursos; ganancias derivadas de la especialización y de las economías de escala; disminución en el coste de las importaciones; e incremento de la competencia. Aunque también hay que tener presente que el comercio tiene importantes efectos redistributivos sobre el bienestar, beneficiando a algunos sectores y perjudicando a otros.

Por otro lado, la mayor interdependencia entre países y el auge de las cadenas de valor ha hecho que el comercio internacional aumente su volatilidad y prociclicidad con el crecimiento económico global.

\section{Evolución reciente de la relación entre comercio y crecimiento a nivel global}

En este contexto de auge de la globalización, el comercio mundial venía creciendo aproximadamente al doble de la tasa del PIB mundial en los años previos a la crisis financiera global, o GFC (pasando la intensidad del comercio, medida como la participación de las importaciones mundiales de bienes y servicios en el PIB, de un $17,5 \%$ en 1986 a un $30 \%$ en 2008, según datos del Banco Mundial). Sin embargo, la relación entre el crecimiento y el comercio pareció cambiar tras la GFC (Figura 1); la elasticidad ingreso del comercio cayó por debajo de la unidad entre 2012 y 2019 (en concreto, pasó de 1,55 entre 1980 y 2007, a solo el 0,93 entre 2012 y 2019, según datos del Fondo Monetario Internacional, World Economic Outlook de octubre de 2021). Si el comercio mundial hubiera continuado expandiéndose de acuerdo con su tendencia previa, habría estado una cuarta parte por encima de su nivel real en 2019.

Hay diversos factores que han podido influir en este cambio en la relación entre el comercio y la actividad mundial. Por tanto, es importante tratar de determinar hasta qué punto se explica por cambios en el crecimiento y la composición de la economía global, o se debe a transformaciones más profundas, como cambios en las fricciones comerciales o la maduración de las cadenas de valor mundiales.

\section{Los efectos composicionales por la participación entre países}

Además de las fluctuaciones en las elasticidades a nivel nacional, los cambios en la elasticidad global también reflejan cambios composicionales en la participación de las importaciones o el crecimiento relativo entre países.

Desagregando la evolución de las importaciones por regiones y países se observa que la debilidad de las importaciones en el periodo posterior a la GFC (2012-2019), respecto a los años previos (1980-2007), ha sido generalizada (Figura 2). Sin embargo, la 

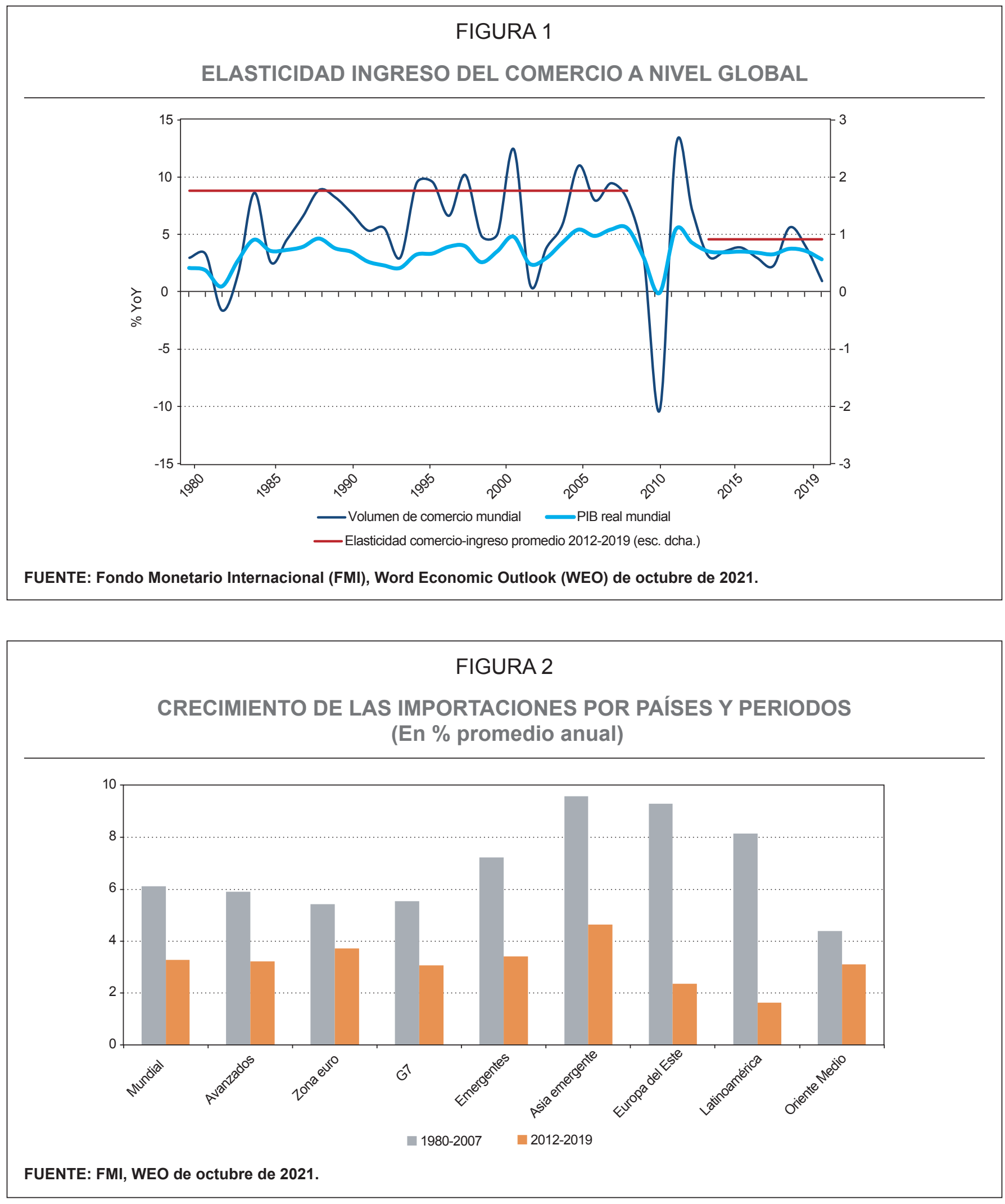
disminución fue mucho más pronunciada en las economías emergentes que en las avanzadas.

De hecho, fijándonos en la elasticidad comercio-ingreso, vemos que en las economías avanzadas esta ha disminuido solo ligeramente durante esos periodos (de 2,1 a 1,7), pero que en los países emergentes su caída ha sido mucho más brusca (de 1,7 a 0,7).

Así, la caída en elasticidad ingreso del comercio global de 0,8 entre el periodo anterior a la GFC y el periodo posterior ( 1,74 antes y 0,96 después) se explicaría: i) casi en su totalidad, 0,78 puntos, por una menor contribución de las economías desarrolladas pero que se explica mayoritariamente por su menor peso en el agregado mundial: su elasticidad comercio-ingreso apenas cayó, pero esta región representó una menor participación en las importaciones mundiales $\mathrm{y}$, fundamentalmente, una caída en el crecimiento del PIB en relación con el promedio mundial; y ii) una contribución de los emergentes prácticamente sin cambios que en cambio esconde una gran disminución de su elasticidad comercial compensada por un mayor peso en la economía mundial.

Luego, casi la mitad de la disminución de la elasticidad del comercio mundial se puede atribuir al cambio en la composición de la demanda relativa entre el grupo de países desarrollados y emergentes, y no a cambios en las elasticidades a nivel nacional.

Los efectos por la composición de la demanda dentro de cada país

Además, no todas las fluctuaciones en las elasticidades a nivel nacional se deben a cambios estructurales en el comercio, sino que en parte se pueden deber también a cambios en la composición de la demanda agregada.

Los componentes de la demanda tienden a tener diferentes intensidades comerciales. En general, la inversión empresarial es más intensiva en comercio que el consumo privado, y este más que el gasto público (Boz et al., 2014). Por tanto, la reciente debilidad de la inversión parece haber jugado cierto papel detrás de la moderación del comercio.

$Y$, aunque puede haber ciertas fuerzas estructurales que han empujado hacia una moderación de la inversión, en gran medida podríamos estar hablando de un fenómeno cíclico y reversible. De hecho, los componentes del PIB suelen evolucionar de forma diferenciada durante el ciclo económico; mientras que la inversión tiende a ser muy procíclica, el gasto público es acíclico o incluso contracíclico. Como resultado, las recesiones inducen una relación entre las importaciones y el PIB diferente a la que existe en épocas expansivas. Adicionalmente, Freund (2009) encuentra que la relación entre el comercio y el PIB cambia durante las recesiones, en parte debido a la reducción de inventarios, y Altomonte et al. (2012) que estos efectos se han amplificado por el desarrollo de la producción mediante cadenas de valor globales.

Por lo tanto, una parte de la moderación del comercio tras la GFC podría explicarse por una debilidad de la inversión, que no tiene por qué ser permanente.

\section{Factores estructurales que están alterando la relación fundamental entre el comercio y la actividad económica}

Algunas fuentes estructurales que han podido alterar la relación fundamental entre el comercio y la actividad económica se materializaron durante el periodo señalado. Estos factores estructurales, que serían los responsables de explicar casi la otra mitad de la caída en la elasticidad del comercio mundial no explicada por los factores de composición, habrían operado principalmente a través de dos canales:

El primero refleja la disminución del apoyo de factores que anteriormente habían contribuido a que el comercio mundial superara el crecimiento de la producción mundial, incluidos los costes de transporte más bajos y la eliminación de las barreras comerciales. Así, los aranceles prácticamente han dejado de caer desde 2010, y hemos visto la aparición de crecientes 
barreras no arancelarias. A ello hay que añadir el incremento en la incertidumbre comercial derivado del estallido de la guerra comercial y tecnológica entre EE UU y China.

El segundo canal, relacionado, aunque más complejo, que puede explicar la reducción observada en la elasticidad renta del comercio tras la GFC refleja la evolución y maduración de las Cadenas Globales de Valor (GVC, por sus siglas en inglés). Los avances en las tecnologías de la información y el transporte y la caída de las barreras comerciales facilitaron una creciente fragmentación de los procesos de producción a lo largo de las cadenas de valor ubicadas en diferentes países, dando lugar a un fuerte aumento del comercio de bienes intermedios. Sin embargo, la participación de la GVC en el comercio se ha estancado desde la GFC.

La literatura ha encontrado varias explicaciones a este proceso de maduración de las GVC. Por un lado, no puede ignorarse el papel de China, constatándose durante los últimos años en este país, que ocupa un papel central en las GVC, un importante proceso de sustitución de inputs importados por producción nacional. Pero, por otro, hay tendencias globales que también lo explican. Constantinescu et al. (2015) argumentan que el shock tecnológico que impulsó el desarrollo de las GVC en los noventa ya ha sido absorbido. Otros autores apuntan a que las nuevas tecnologías de producción, más intensivas en capital y menos en factor trabajo, han reducido los incentivos al fragmentar la producción en el caso de que una motivación para ello fuesen los ahorros de ubicar la producción donde los trabajadores eran más baratos. Estas tecnologías (la robotización, impresoras 3D, plataformas digitales) habrían impulsado una tendencia hacia la regionalización de las GVC (Baldwin, 2011).

No obstante, un aumento de la regionalización, la tendencia a cadenas de producción más cortas, y cierto retorno a las economías avanzadas de actividades anteriormente deslocalizadas, no tiene por qué ser incompatible con una elasticidad del comercio elevada, si domina el efecto de reducción de costes y de impulso de las mejoras competitivas, lo que alienta la innovación, y a su vez da lugar a la comercialización de nuevos bienes y servicios, aumentando la expansión de las GVC y del comercio (Antràs, 2020). Además, estas nuevas tecnologías ayudan a que las empresas pequeñas puedan salir más fácilmente más allá de sus mercados locales.

\section{La importancia del sector servicios en el comercio}

Tradicionalmente el sector servicios ha sido menos abierto al comercio, pero los datos muestran no solo que las importaciones mundiales de servicios han aumentado de manera constante durante los últimos 30 años, sino que la participación de las importaciones de servicios dentro del total también ha aumentado (el comercio de servicios ha crecido un $60 \%$ más rápido que el comercio de bienes durante la última década).

Además, problemas en la correcta medición del comercio de servicios enmascaran su importancia en el comercio internacional. Así, según cifras de balanza de pagos, aunque los servicios representan más del $70 \%$ del PIB mundial, solo suponen alrededor del $20 \%$ del comercio mundial. Sin embargo, la función de los servicios como insumo en la producción manufacturera, a menudo denominada servicificación de la manufactura, es sustancial. Los servicios crean aproximadamente un tercio del valor que se destina a los bienes manufacturados comercializados. I $+D$, ingeniería, ventas y marketing, finanzas y recursos humanos permiten que los productos lleguen al mercado. Así, según bases de datos como TiVA de la OCDE, en términos de valor agregado y teniendo en cuenta los servicios incorporados en los bienes exportados, los servicios representan el $40 \%$ del comercio mundial, a lo que habrá que unir los contabilizados directamente como importaciones de servicios para llegar a que más del $65 \%$ del comercio internacional es de servicios.

Con todo este análisis previo de la relación global entre comercio y crecimiento, pasaremos a analizar 
el caso español y si los cambios pos-GFC nos permiten ser o no optimistas sobre la contribución futura del comercio exterior español al crecimiento. Para ello pasaremos ahora a analizar los datos de exportaciones de bienes y servicios (EBBSS) e importaciones de bienes y servicios (IBBSS).

3. España. El distinto comportamiento del sector exterior antes y después de la crisis de 2009-2012. Los datos de España y de países avanzados

Durante los años 2000-2007, correspondientes al periodo anterior a la GFC de 2008, las exportaciones españolas en volumen mostraban un relevante crecimiento anual promedio del $4,8 \%$, pero crecían sustancialmente menos que las exportaciones de países avanzados (con un crecimiento anual promedio del 7,1 \%). Mientras las importaciones aumentaban al mismo ritmo en España que en avanzados (el $7 \%$ ), nuestro país generaba un desequilibrio exterior que fue un detonante de la crisis.

Para simplificar la presentación de datos, hemos tomado la media del periodo 2014-2019 para compararla con 2000-2007 (Tabla 1). El cambio desde 2012 es espectacular, hemos pasado a que nuestras exportaciones crezcan bastante más que en los países avanzados y a que aumenten dos veces más que el PIB español. Es verdad que la tasa media de nuestras importaciones es solo ligeramente superior a la de las exportaciones, pero es un cambio radical respecto a la situación precrisis. El crecimiento del PIB se moderó, pero es similar a los países de nuestro entorno. Los datos tienen todavía una lectura más positiva si tenemos en cuenta que disminuye el contenido de importaciones incorporado a nuestras exportaciones.

En resumen, en la fase de recuperación y crecimiento desde la «gran crisis» el sector exterior contribuyó positivamente al crecimiento, como se observa en la Tabla 2 esto fue tanto por crecimiento de exportaciones como por menor crecimiento de importaciones.

\section{TABLA 1}

TASAS DE CRECIMIENTO EN VOLUMEN DE EXPORTACIONES, IMPORTACIONES, Y PIB EN PAIISES AVANZADOS Y EN ESPAÑA

Volumen de exportaciones de bienes y servicios

\begin{tabular}{|c|c|c|}
\hline & $2000-2007$ & $2014-2019$ \\
\hline $\begin{array}{l}\text { Exportaciones España } \\
\text { Tasa de variación anual (\%) }\end{array}$ & 4,8 & 4,1 \\
\hline $\begin{array}{l}\text { Exportaciones países avanzados } \\
\text { Tasa de variación anual (\%) }\end{array}$ & 7,1 & 3,2 \\
\hline
\end{tabular}

Volumen de importaciones de bienes y servicios

Importaciones España

Tasa de variación anual (\%)

$$
\begin{array}{cc}
2000-2007 & 2014-2019 \\
\hline 7,0 & 4,4
\end{array}
$$

Importaciones países avanzados $\quad 7,0$ 3,6

Tasa de variación anual (\%)

PIB en términos reales
PIB España

Tasa de variación anual (\%)

PIB países avanzados

Tasa de variación anual (\%)

FUENTE: Instituto Nacional de Estadística (INE) y FMI.
Como consecuencia de estas tasas de crecimiento mencionadas, las exportaciones de bienes pasaron del $15 \%$ del PIB en 2009 a alcanzar un peso máximo del $24 \%$ desde 2017 a 2019, nueve puntos más. lgualmente, el peso de las exportaciones de servicios en el PIB aumentó de un 7,7 \% en 2009 a un nivel máximo del $11 \%$ desde 2016 a 2019. Por consiguiente, el peso de las exportaciones conjuntas de bienes y servicios sobre el PIB se incrementó en doce puntos, desde un $23 \%$ en 2009 hasta los máximos alcanzados desde 2017 a 2019 del 35 \% del PIB. La estabilización de estas ratios a partir del 2017 puede ser consecuencia de cambios en el comercio internacional o de un cambio que responda a factores exclusivamente españoles. Es importante analizarlo para poder realizar alguna hipótesis 
TABLA 2

CONTRIBUCIÓN DE LA DEMANDA EXTERNA AL CRECIMIENTO REAL DEL PIB

Contribuciones del sector exterior a la tasa de variación anual del PIB (En puntos porcentuales)

\begin{tabular}{lcc} 
& $2000-2007$ & $2014-2019$ \\
\cline { 2 - 3 } Demanda externa & $-0,8$ & 0,1 \\
Exportaciones & 1,3 & 1,4 \\
Importaciones & $-2,1$ & $-1,3$
\end{tabular}

FUENTE: INE.

sobre el comportamiento esperado del sector exterior en la recuperación pos-COVID-19.

Por otro lado, las exportaciones españolas también han tendido a mejorar su diversidad geográfica, aunque el peso de nuestras exportaciones de bienes hacia Europa sigue siendo enorme, situándose en 2019 en el $64 \%$ de total de las exportaciones de bienes.

\section{Las causas y la narrativa del buen comportamiento del sector español después de la GFC}

A raíz de las crisis de 2009 y de 2012 nuestro país sufrió un importante ajuste del empleo y de los costes salariales. La salida de la crisis pasó por una contención forzada de la demanda interna y por la implementación de reformas estructurales especialmente en el mercado laboral, que generaron importantes ganancias de competitividad, tanto vía precio como vía factores de índole cualitativo, posibilitando la corrección de los desequilibrios externos por cuenta corriente.

De modo que la economía española después de registrar un abultado déficit por cuenta corriente, que alcanzó el 9,4 \% del PIB en 2007 y el 8,9 \% en 2008, pasó a alcanzar superávit corriente en 2012, del 0,1\% del PIB, manteniéndose desde entonces en superávit hasta la actualidad.

La narrativa es que la ganancia de competitividad vía costes salariales frente a nuestros socios de la zona euro permitió mejorar nuestra competitividad externa, promoviendo la internacionalización de nuestras empresas. En términos de costes laborales unitarios, estos niveles son menores que los de nuestros socios europeos, y su evolución relativa, que había empeorado hasta 2007, después mejoró revirtiendo el deterioro previo.

Esta mejora es también clara tomando solo los costes laborales unitarios en las manufacturas, donde también aplica la ventaja de salarios más bajos. Dada esta diferencia salarial y el mejor track record de reformas estructurales en España, no contemplamos un empeoramiento de las ventajas competitivas españolas frente a la Unión Europea (UE). Solo falta por saber si esta mejora desde 2007 ya ha agotado sus efectos en el sector exterior, aunque no hay evidencia.

En cuanto a la productividad real, definida como el valor añadido por persona empleada a tiempo completo, se encuentra que España ha conseguido evoluciones algo inferiores a Francia y a Alemania desde la creación del euro, y claramente superiores a Italia. Pero, y esta es la clave de las ganancias de competitividad, esa productividad menos los costes salariales deflactados por el deflactor del valor añadido bruto (VAB) (compensación real media por empleado) es positiva y más alta en España, incluso desde 2004. Desde la crisis solo España y Alemania presentan esta ganancia, mientras que, en Italia y Francia, desde la crisis crecen más las compensaciones que la productividad (Figura 3). Luego España consigue mejoras de competitividad por una reducción de la participación de las rentas salariales en ese VAB. Es lo que podríamos llamar una devaluación interna al área monetaria. Este tema será clave mantenerlo para conseguir más contribución neta positiva del comercio.

También en cuanto a las reformas del mercado de productos y de factores, la OCDE en 2018 nos sitúa 
FIGURA 3

EVOLUCIÓN DE LA PRODUCTIVIDAD LABORAL REAL VERSUS COSTES LABORALES DEFLACTADOS POR EL DEFLACTOR DEL VAB

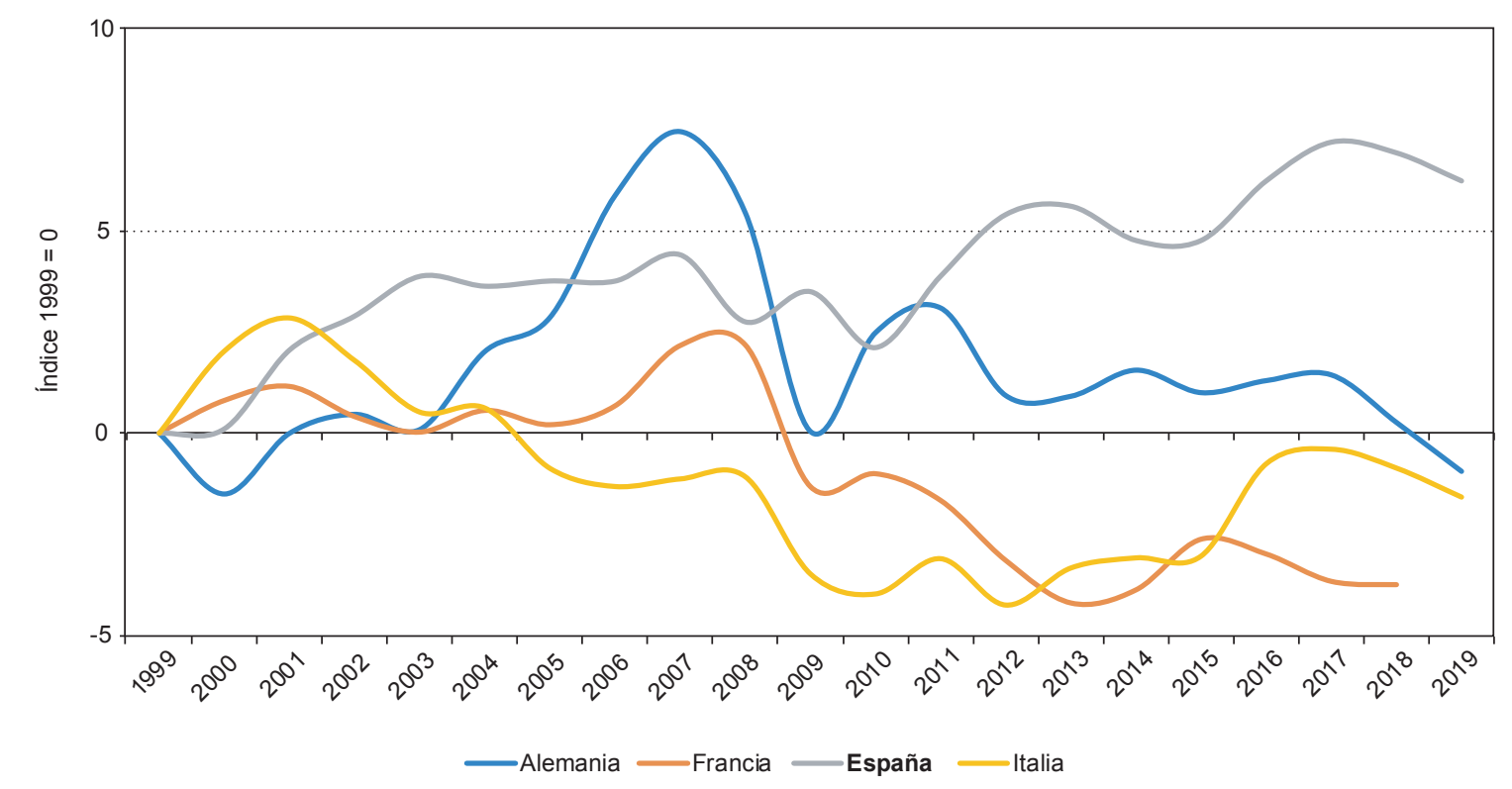

FUENTE: Organización para la Cooperación y el Desarrollo Económicos (OCDE).

entre los mejores, por lo tanto, y de manera muy simplificada parece que las variables que explicaron la mejora del comportamiento de nuestras exportaciones - más allá del crecimiento de los países a los que exportamos - deberían sostener en los próximos años una buena tendencia. Según los indicadores de la OCDE el entorno regulatorio en nuestro país ha mejorado incentivando la competencia en nuestro mercado de productos.

Por último, también ha habido ganancias de competitividad vía tipo de cambio efectivo nominal que contribuyeron a nuestras mayores exportaciones, aunque la sensibilidad de estas al tipo de cambio suele ser baja. En esta variable no contemplamos grandes cambios en los próximos años, y dado también su menor poder explicativo la hemos excluido en el resto de nuestro análisis.
Todos estos indicadores de mejora de competitividad vía precio y vía otros factores de índole cualitativa, como un mejor marco regulatorio, han posibilitado la favorable evolución de nuestro sector exterior en los últimos años. No se ven cambios de tendencias en dichos indicadores de competitividad —si bien en el año 2019, aumentan más compensaciones que productividad-, y las ganancias potenciales en términos de comercio exterior no tienen por qué haberse agotado si como se señala se parten de ventajas absolutas de costes. Aunque no se observan cambios de tendencia -es verdad que los años 2018 y 2019 fueron muy especiales para el comercio internacional por la guerra comercial-, se podría concluir provisionalmente que España continúa teniendo una buena competitividad externa que debería permitir una buena contribución al crecimiento pos-COVID-19. También sería importante 
FIGURA 4

PIB REAL E IMPORTACIÓN DE BIENES Y SERVICIOS EN ESPAÑA Y LA UNIÓN EUROPEA
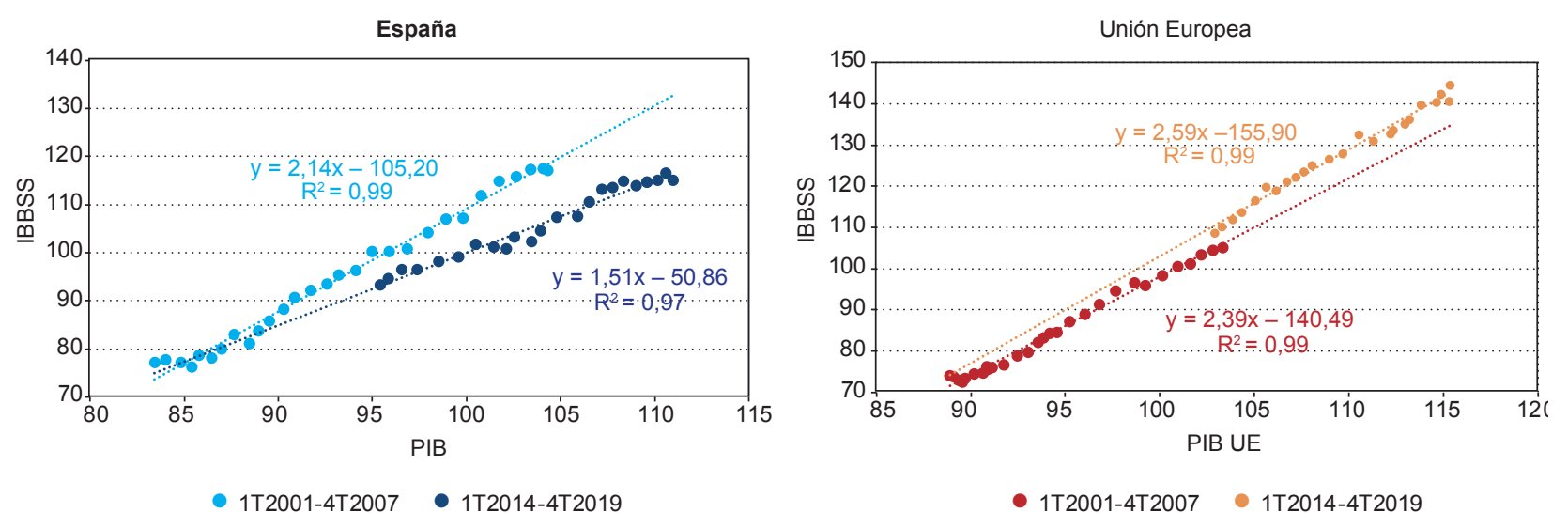

FUENTE: INE y Eurostat.

reforzar la idea de que no podemos perder las ventajas competitivas en los próximos años con políticas laborales y regulatorias inapropiadas que se desacoplen de la evolución de la productividad.

\section{Elasticidades de exportaciones y de} importaciones de España y de nuestros socios europeos tras la GFC; implicaciones para la contribución futura del sector exterior

En este apartado se realiza un análisis de la relación existente entre el sector exterior y la evolución del PIB y sus principales variables explicativas, tanto para España como para la UE, y compararemos cómo han cambiado entre antes de la crisis de 2008-2013, Gran Crisis Financiera (GFC), y el periodo 2014-2019. Es decir, utilizando como variable de referencia las importaciones de bienes y servicios, vamos a estudiar su relación con el PIB y sus principales agregados macroeconómicos: consumo privado, inversión y exportaciones. Con este análisis tratamos de identificar si se han producido cambios estructurales y si para cada uno de los componentes se producen cambios en su intensidad de importaciones. El análisis aquí realizado, para establecer el comportamiento conjunto de estas variables, es un análisis estadístico sencillo que busca la relación directa existente entre ellas y que se utiliza como un argumento más, y no único, para corroborar la tesis expuesta en este artículo. Solamente se ha realizado un análisis más econométrico para la relación PIB e importaciones.

Vamos a estudiar el periodo de 2001 a 2019 subdividido en dos tramos, de forma que por lo general no vamos a tener en cuenta el periodo 2008-2013, los años de la GFC en España. Utilizando estos dos periodos evaluaremos cómo la GFC supuso para España cambios estructurales en sus relaciones comerciales. Este cambio estructural, entre las importaciones y el PIB, se puede observar a simple vista en la representación de la Figura 4 de ambas variables en niveles. No obstante, para comprobar que la relación entre importaciones y PIB no es espuria, se han realizado los correspondientes test de causalidad, confirmando que no podemos rechazar una causalidad entre las variables en ambos sentidos. 
Asimismo, se ha realizado un modelo econométrico sencillo con variables dummy, donde se ha constatado el cambio estructural en los periodos citados (los cálculos se incorporan en Anexo). Además, haremos la comparativa con la Unión Europea, para ver si también ha experimentado cambios estructurales tras la GFC y si los cambios diferenciales permiten aventurar un mejor comportamiento de nuestro sector exterior con la UE.

\section{Relación PIB e importaciones}

La relación clave es si ha habido un cambio estructural en cuanto a la relación entre las importaciones de bienes y servicios (IBBSS) españoles y la evolución de su PIB. Tal y como se puede observar en la Figura 4 la relación entre estas dos variables es distinta antes y después de la crisis. Tras la GFC, la economía española no solo se hace menos intensiva en importaciones, sino que, además, la pendiente de la curva disminuye haciéndose menos elásticas las importaciones al crecimiento del PIB. De hecho, si tomáramos los tres últimos años esta elasticidad seria todavía más baja. Esto contrasta con lo sucedido para la UE, donde el cambio es mínimo, y se ha producido en sentido contrario, más importaciones por unidad de PIB. Para contrastar este análisis sencillo de regresión hemos estudiado si hay cambio estructural mediante la introducción de una variable dummy. La conclusión es que sí y el análisis realizado se incorpora como Anexo a este trabajo.

En definitiva, tras la GFC, la economía española relativa al conjunto de la UE ha pasado a ser menos intensiva en importaciones $\mathrm{y}$, además, más inelástica al PIB, e incluso en los últimos años la diferencia aumenta más a favor de España. Por lo tanto, a igualdad de crecimiento económico entre la UE y España, en la recuperación pos-COVID-19, en España crecerán menos las IBBSS y la contribución al crecimiento del comercio internacional tenderá a aumentar (aunque stricto sensu sería con respecto al comercio total, esto parece ya visible en los flujos bilaterales).
Una vez que hemos establecido cómo ha cambiado para España la relación entre el PIB y las importaciones de bienes y servicios, es necesario establecer por qué se ha producido dicho cambio. Para ello vamos a entrar en detalle, analizando cómo se ha visto afectada la relación entre las importaciones y el resto de componentes del PIB: exportaciones de bienes y servicios (EBBSS), la formación bruta de capital (FBC) y consumo final de los hogares (CFH).

\section{Relaciones entre IBBSS y EBBSS}

Vamos a analizar los cambios en estos dos periodos y por lo tanto las nuevas elasticidades pos-GFC 2014-2019 para exportaciones (EBBSS) y formación bruta de capital (FBC) y comprobaremos tambien que no hay cambios significativos en las mismas cuando analizamos solo el periodo 2017-2019 (estos cálculos no se incluyen en la parte publicada de este trabajo). Como sabemos que puede haber más factores explicativos, comprobaremos a continuación cómo el análisis con datos de valor añadido confirma o no confirma estas hipótesis. Intentar con los datos de contabilidad nacional algo más concluyente no lo hemos visto en la literatura, y los cálculos que hacemos no son concluyentes. Por eso se presenta este análisis simple.

Comenzamos el analisis de sensibilidad por componentes con la relación importaciones/exportaciones (Figura 5). Básicamente, cambios en la interrelación entre exportaciones e importaciones indican un cambio en la aportación neta del sector exterior al crecimiento del PIB, independientemente de las casusas que lo expliquen. Parece que tras la GFC se ha producido una mejora en la posición competitiva de la economía española, sobre todo con respecto a la UE, donde se encuentran sus principales socios comerciales.

Se puede observar gráficamente cómo, tras la GFC, la relación entre exportaciones e importaciones cambió notablemente en el caso de la economía española, tanto en nivel como en pendiente (elasticidad), mientras que en la UE no se registró prácticamente ningún 
FIGURA 5

\section{RELACIÓN IMPORTACIONES Y EXPORTACIONES DE BIENES Y SERVICIOS EN ESPAÑA Y UNIÓN EUROPEA}
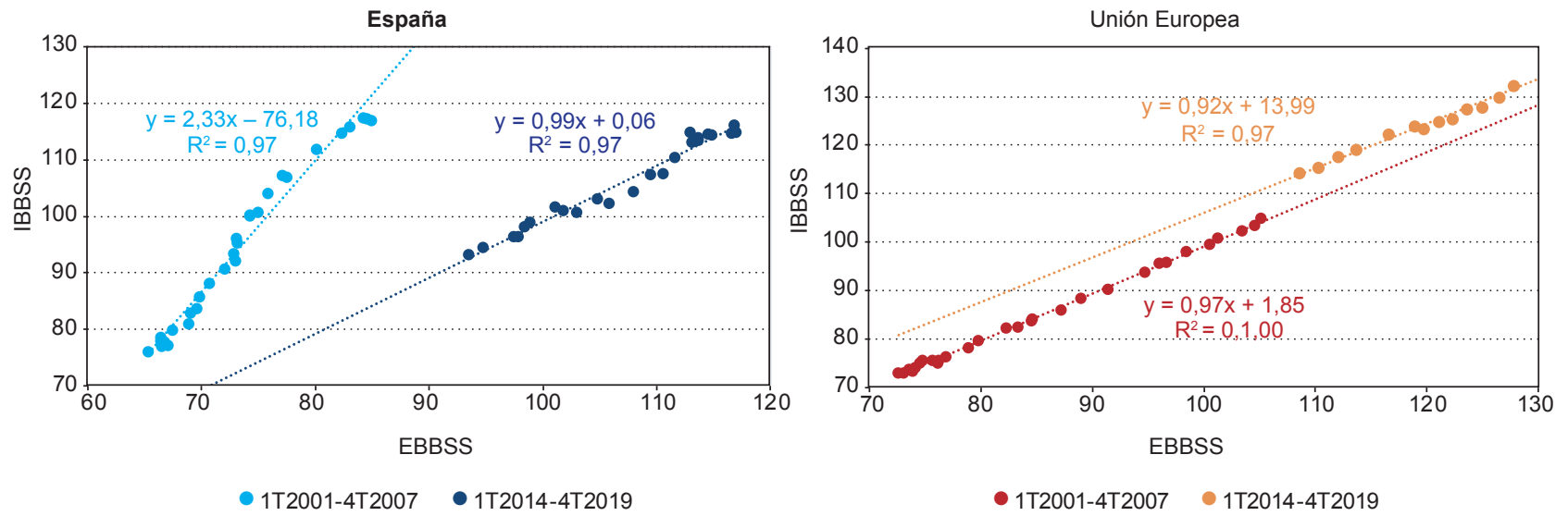

FUENTE: INE y Eurostat.

cambio. Estos cambios supusieron para la economía española no solo un descenso significativo de las importaciones de bienes y servicios por unidad de exportación, sino también un importante descenso de la elasticidad de las importaciones con respecto a las exportaciones. Teniendo todo esto en cuenta, podemos concluir que si crecen mucho nuestros mercados claves de exportación -la Unión Europea - la contribución del sector exterior será mayor que en el pasado, porque se genera más valor añadido español por unidad exportada y menos valor de importaciones incorporadas ${ }^{1}$.

Tras la GFC España converge a una elasticidad similar a la de la UE. Por lo tanto, a igual crecimiento de exportaciones, en ambas zonas las importaciones de bienes y servicios serán similares.

\footnotetext{
${ }^{1}$ Esto se explica en gran parte por el fuerte crecimiento diferencial de la exportación de servicios y se analizará más detenidamente en otro apartado donde se presentan los datos de comercio por valor añadido de la OCDE conocidos como TiVA.
}

\section{Relaciones entre IBBSS y FBC; IBBSS y construcción y bienes de equipo (BBE)}

\section{Relación entre IBBSS y FBC}

En el caso de la FBC, vemos en la Figura 6 que con posterioridad a la crisis aumenta la proporción de IBBSS por FBC; es básicamente un salto hacia menor demanda doméstica. Este hecho se explica porque en el periodo anterior la inversión en construcción, que no requería tantos insumos externos, era el componente que más crecía, frente a los bienes de equipo. Por su parte, la sensibilidad de las importaciones a la inversión experimenta una leve mejoría, se requiere menos IBBSS por unidad de FBC (cambio de pendiente en la curva). En este caso, si calculamos las elasticidades en el subperiodo 2016-2019 el cambio es todavía más positivo, pues pasa de 0,85 a 0,70 .

No obstante, entrando en detalle de las causas de este cambio en la inversión debemos tener en cuenta 


\section{FIGURA 6}

\section{RELACIÓN IMPORTACIONES Y FORMACIÓN BRUTA DE CAPITAL EN ESPAÑA}

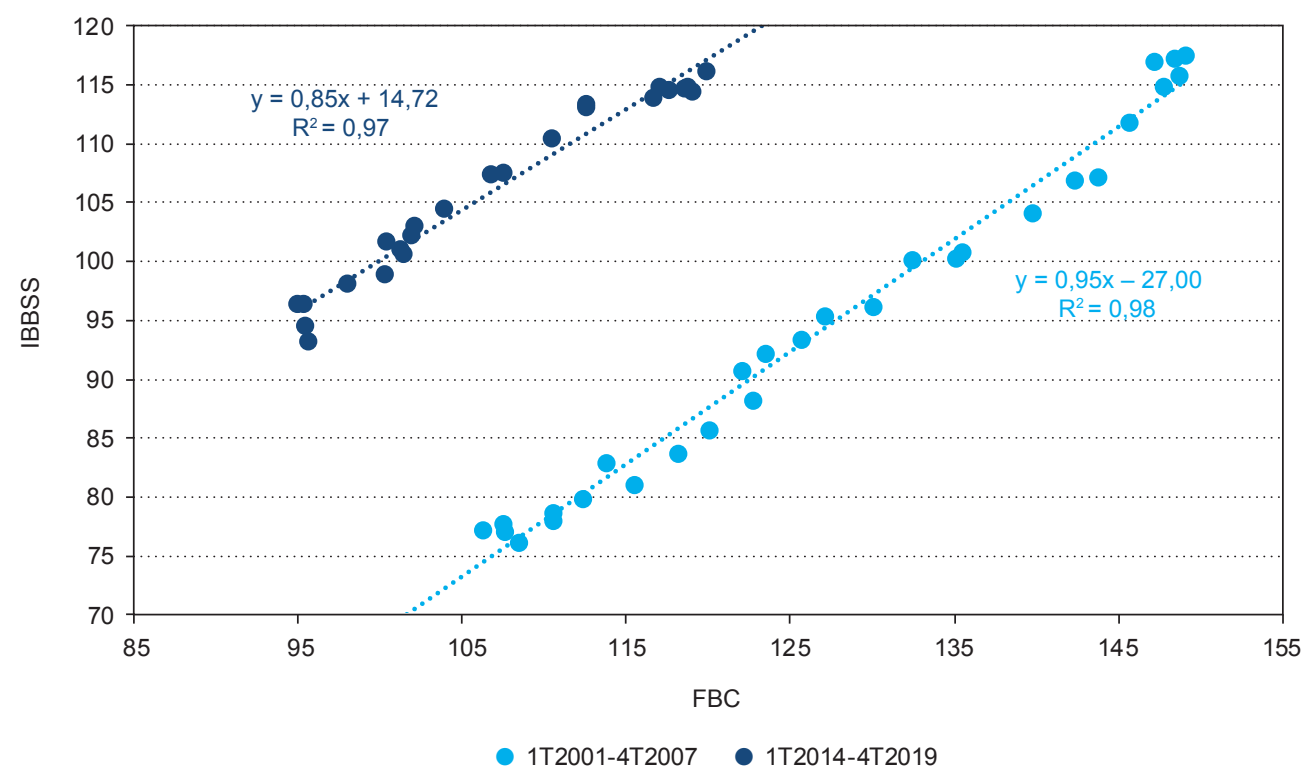

FUENTE: INE.

el fuerte cambio estructural por la caída del peso de la construcción en nuestro país sobre la FBC, pasando del $65 \%$ en el primer periodo a menos del $50 \%$, frente a un aumento de la participación de la formación de los bienes de equipo (FBBE), del $25 \%$ al $31 \%$. En el caso de inversión en bienes de equipo, podemos observar primero; que solo en 2019 alcanzamos los niveles precrisis y que lo hace con la misma ratio IBBSS que en 2007; segundo, que la pendiente de la curva o la elasticidad de las IBBSS a los bienes de equipo cae mucho en el segundo periodo (2014-2019), lo que implicaría un aumento del valor añadido domestico (VAD) por bien de equipo producido (Figura 7).

Pero es en la inversión en construcción donde nos encontramos con el cambio más importante porque es el componente que más ha reducido su peso en el PIB, y que sigue lejos de su nivel pre-GFC, y donde se ve el salto de nivel más alto en la ratio IBBSS/FBC en el primer año pos-GFC, aunque la elasticidad o relación, a partir de entonces, permanece estable y similar al periodo precrisis.

En cuanto a la comparación con la UE, de la relación poscrisis entre la inversión y las importaciones podemos ver que la inversión en España no solo requiere menos importaciones, sino que, además, es más inelástica (Figura 8). Y, por lo tanto, de mantenerse esta tendencia, a igualdad de crecimiento de la inversión en ambas zonas, España importaría relativamente menos, generando más valor añadido interno. Intentaremos comprobar esta hipótesis en el siguiente subapartado.

\section{Relación entre IBBSS y CFH}

Por último, el consumo final de los hogares no ha mostrado un cambio significativo en su relación con las 

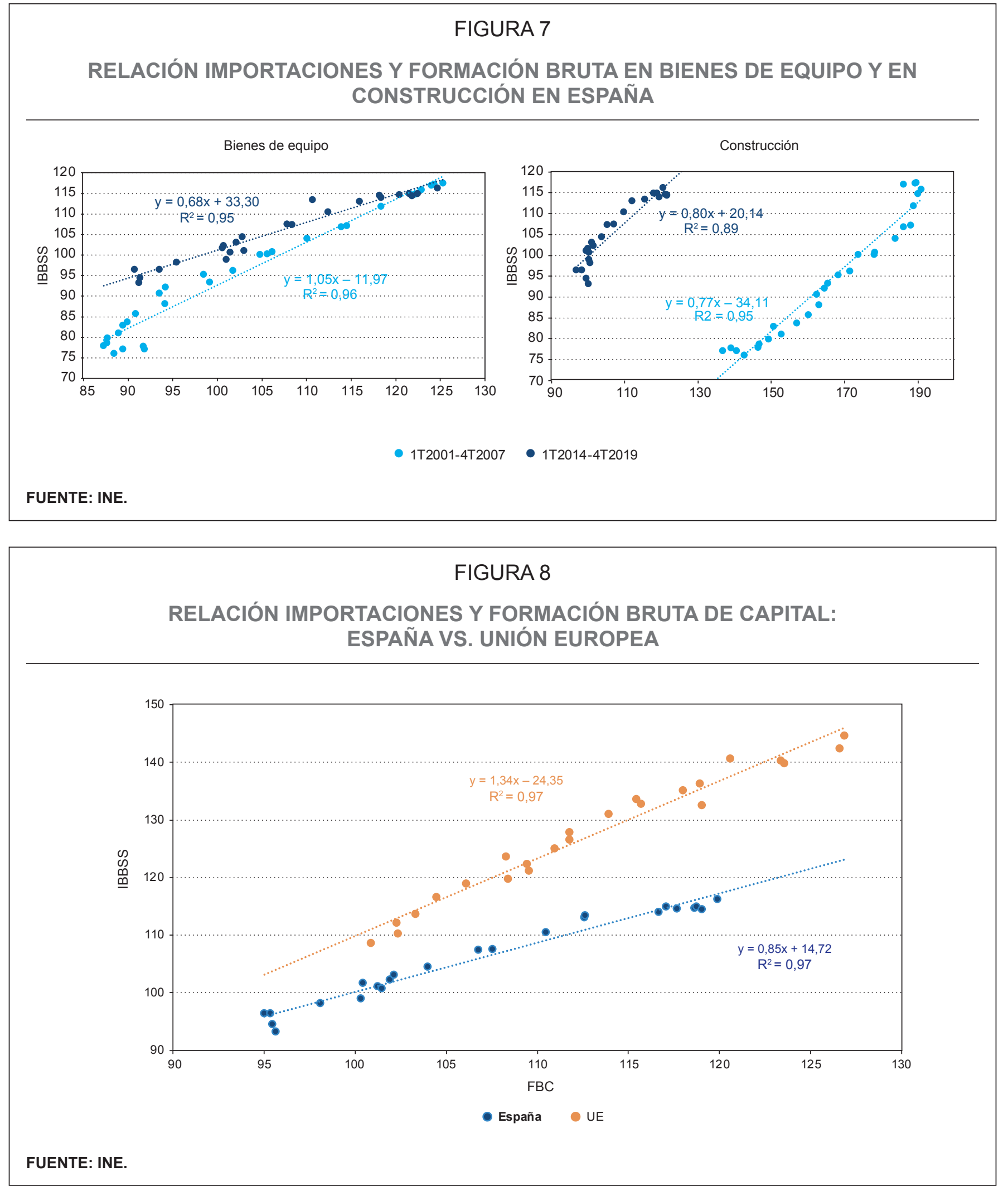


\section{FIGURA 9}

\section{RELACIÓN IMPORTACIONES Y CONSUMO FINAL DE LOS HOGARES EN ESPAÑA, Y COMPARACIÓN ESPAÑA Y UNIÓN EUROPEA}
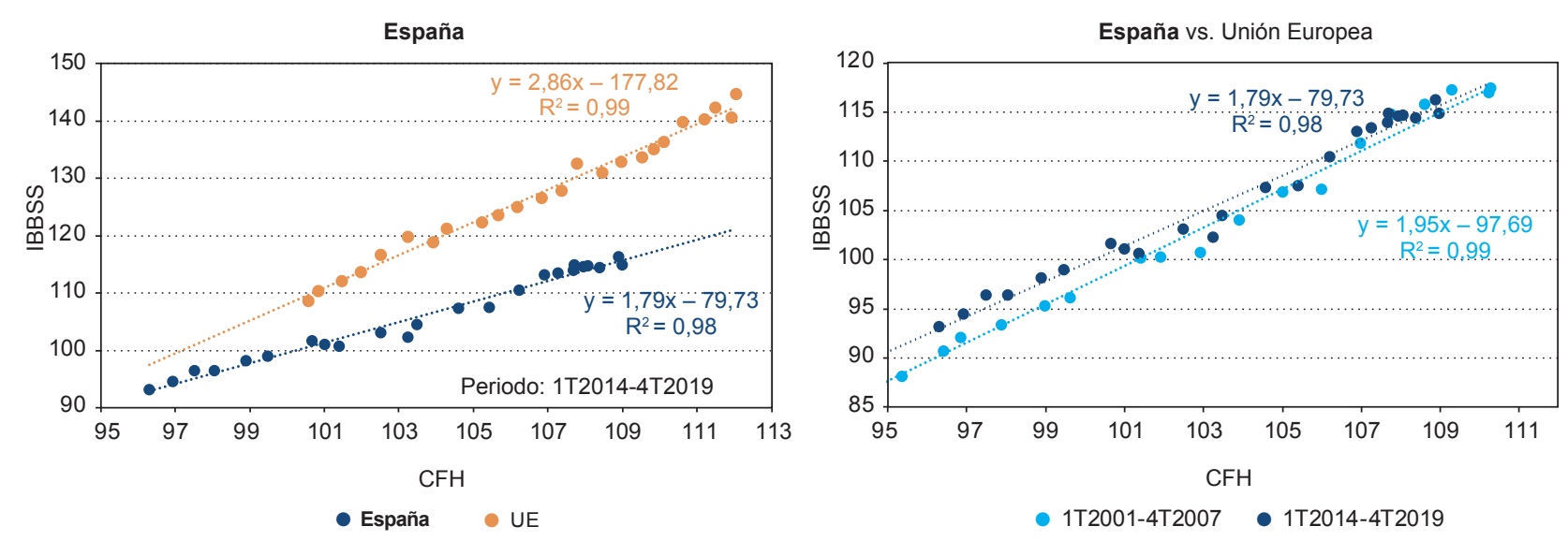

FUENTE: INE.

importaciones. Al compararlo con la UE, en la economía española el consumo final de los hogares muestra ser menos intensivo en importaciones y registra una menor elasticidad ante las mismas (Figura 9).

\section{Relación entre exportaciones y zonas geográficas}

Por otro lado, atendiendo a la influencia geográfica sobre las exportaciones de bienes y servicios, podemos observar que, tras la GFC, se produce un salto con más exportaciones por unidad de PIB europeo y que aumenta la elasticidad de nuestras exportaciones al crecimiento europeo. En lo que respecta a las economías emergentes, que crecen mucho más que la UE, el cambio es el contrario. Se produce un salto hacia menores EBBSS por unidad de PIB emergente que la que hubiese habido de continuar esa tendencia, es decir, menor elasticidad. Esto es compatible con lo analizado en el apartado dos, pues equivale a una menor elasticidad de importaciones de los países emergentes.
Como consecuencia, en los últimos años aumentaron más nuestras exportaciones a Europa durante la crisis (en volumen al menos). Sin embargo, tras la crisis, la elasticidad es algo mayor que antes de ella. Por último, se observa que en los últimos años se reduce la elasticidad, lo que puede ser debido a la guerra comercial y a menores importaciones.

Como se ha señalado, en este subapartado se ponen de manifiesto relaciones entre las importaciones y el PIB que son explicativas y otra respecto a las IBBSS y los componentes del PIB y, aunque el dato analizado es de IBBSS totales y no por tipología de bienes o servicios, dada la consistencia temporal de tipología de bienes en la composición de nuestras exportaciones, se podría concluir que también tiene poder explicativo.

Con este análisis tan sencillo se ve solamente el cambio porcentual en las tasas de crecimiento, pero al compararlas con la UE arroja luz tanto sobre la consistencia de esas relaciones como de los efectos sobre el crecimiento pos-COVID-19 que tendría 
el mantenimiento de las mismas. Estas tendencias muestran que a igualdad de crecimiento de la Unión Europea y España mejorará el saldo exterior español. Además, dada la menor caída de rentas europeas, es de esperar una recuperación del consumo más alto en Europa, lo que dado las elasticidades de las importaciones vistas supone también una mejora relativa para la aportación neta del sector exterior de la economía española.

\section{Análisis de los datos de valor añadido de exportaciones e importaciones de España y de los principales socios exteriores ${ }^{2}$. Resumen ejecutivo}

Para este apartado, utilizaremos la base de datos de comercio en valor añadido, Trade in Value Added (TiVA), estadísticas de la Organización Económica para la Cooperación y el Desarrollo Económicos (OCDE). Esta base de datos ha sido actualizada en noviembre de 2021, y el espectro temporal de la información de la que disponemos varía para ciertos datos de 2005 a 2018. Las cifras en valor son brutas nominales en dólares americanos a precios de mercado, excepto los datos explícitos de valor añadido que se computan de acuerdo con la contabilidad nacional en nominales a precios básicos, es decir, antes de impuestos.

Los datos TiVA de exportaciones no coinciden con los de la contabilidad nacional por que se realizan una serie de correcciones para evitar incongruencias y mejorar la información sobre el valor añadido; en la cifra total TiVA de exportaciones brutas no figuran las reexportaciones y el valor de los bienes frente a los servicios se presenta de manera distinta al valor del bien exportado en contabilidad nacional. En TiVA es el valor a salida de fábrica y no el valor FOB (Free On

\footnotetext{
2 Se agradece la colaboración en la elaboración de las figuras y comentarios de Daniel Montero Rivas, becario de la Dirección de Estudios.
}

Board) de embarque y se consideran servicios exportados al valor de los servicios de transporte hasta puerto y todos los servicios necesarios para la producción y exportación del bien.

El objetivo de este apartado es comprobar si la recuperación española desde 2014 implica cambios diferenciales en el comportamiento del sector exterior, tanto en valor añadido entre España y nuestros principales socios, los países grandes de la Unión Europea como con China y el resto del mundo. También se pasa revista a los principales sectores exportadores españoles y a su relación de exportación bruta y Valor Añadido (VA). El fin último es ver si esas tendencias permiten extraer hipótesis y conclusiones sobre cómo contribuirá a la recuperación en curso el sector exterior.

En primer lugar, es claro que durante el periodo 2005-2018 España mejoró no solo sus EBBSS a sus principales socios sino el valor añadido doméstico incorporado en ellas. Dada la continuidad e incluso la reducción de la elasticidad de las importaciones a las EBBSS en 2016-2019, tal como se presentó en el apartado anterior, es previsible que en los próximos años siga mejorando el comportamiento del sector exterior por dos razones: en primer lugar, porque esta mejora en la relación de importaciones necesarias para exportar no es visible en nuestros principales socios comunitarios ni en la Unión Europea como un todo, y dado que la UE es nuestro primer socio esa mejora relativa aplica al $70 \%$ de nuestro sector exterior. En segundo lugar, porque la mejora es en gran parte relevante por el crecimiento relativamente más alto de las exportaciones de servicios turísticos españoles.

También es importante destacar que el valor añadido español en las importaciones de bienes finales (demanda final de importaciones de España), que excluyen los consumos intermedios reexportados procedentes del resto del mundo, también aumentó más que el valor de dichas importaciones brutas. Esta mejora en el periodo 2005-2015 también explica una mejor situación de nuestro sector exterior en 
cuanto a contribución al crecimiento. Aunque mejoramos en nuestra aportación de valor añadido en las exportaciones y en las importaciones de nuestros principales socios, este no es el caso con el resto del mundo donde crecen nuestras exportaciones e importaciones lo mismo que nuestro VA, probablemente porque hay menos servicios incorporados.

En conclusión, teniendo en cuenta que no vemos grandes cambios en las elasticidades de las importaciones ante los crecimientos de consumo, inversión y exportaciones en nuestro país y en nuestros principales socios, y que algunos cambios lo hacen a mejor, deberíamos concluir que el sector exterior seguirá siendo clave en la recuperación pos-COVID-19.

Si bien no se esperan las correcciones tan fuertes - por positivas - como las que hubo en el periodo 2013-2019, dado que fueron consecuencia de la reducción de grandes desequilibrios y de devaluaciones competitivas que no se contemplan ahora. Por último, una salvedad, los años 2018 y 2019 deben leerse con precaución dado los efectos sobre el comercio internacional de la guerra comercial chino-americana y sus potenciales consecuencias en el largo plazo. En cualquier caso, con la continuidad de las tendencias pre-COVID-19 del sector español, somos de la opinión de que la economía española contará con un sector exterior que no lastrará el crecimiento en los próximos años, sino que aportará valor añadido superior al de recuperaciones anteriores. Veamos con más detalle por qué se extraen estas conclusiones.

\section{Comparación entre España y nuestros socios europeos de la evolución del valor añadido de las EBBSS}

A continuación, se realiza un análisis detallado. Veamos en primer lugar cómo España mejora en cuanto al valor añadido neto más que sus socios entre 2005 y 2018, y cómo esta mejora es mayor frente a China y es especialmente importante en el sector servicios, que como se señala, es el de mayor crecimiento.
Si comparamos el valor añadido foráneo (VAF) de nuestras exportaciones brutas, es decir, la parte de nuestras exportaciones brutas que ha sido previamente importada y es de origen extranjero, podemos observar en la Figura 10 que España es el único país de los cuatro grandes de la zona euro que, en 2018, incorpora menor valor añadido foráneo que en 2005.

Cuando descendemos a analizar bienes industriales y servicios por separado, vemos que, durante el periodo analizado, el sector servicios ha tendido a incorporar mayor valor añadido doméstico —-menor contenido importado - mientras que en bienes industriales ha ocurrido lo contrario (Figura 11).

Si hacemos una comparación de la evolución del VA en bienes industriales entre España y los grandes países de la zona euro, vemos que entre 2005 y 2018, la caída del Valor Añadido Doméstico en España ha sido mucho menor, es decir, el que experimenta el menor crecimiento de Valor Añadido Foráneo en sus exportaciones entre el periodo $(+5,2 \%)$, versus $+15,1 \%$ Alemania, $+18,7 \%$ Francia o $+11,1 \%$ Italia. Esto es aún más claro en servicios, ya que España es el único que se sitúa en el año 2018 en un porcentaje de VAF en servicios sobre exportaciones brutas menor que en 2005 (13,2\% en 2005 vs. $11,9 \%$ en 2018).

Analizando la situación por sectores se encuentra que la evolución agregada -más positiva en España que en los tres grandes de la zona euro en términos de VAFse explica en parte por el hecho de que, algunos de los sectores de exportación que más crecen desde 2015 son aquellos que tradicionalmente incorporan mayor valor añadido doméstico. Estos son los servicios, turísticos y no turísticos, la alimentación y los bienes de equipo. Esta mejora estructural del sector exterior debería continuar ayudando a la recuperación pos-COVID-19.

Respecto a uno de los principales sectores exportadores, el automóvil, nuestra generación de valor añadido doméstico a nuestras exportaciones era el menor de los cuatro grandes en 2005 y muy alejado del segundo, Francia. Ahora en 2018 superamos a 


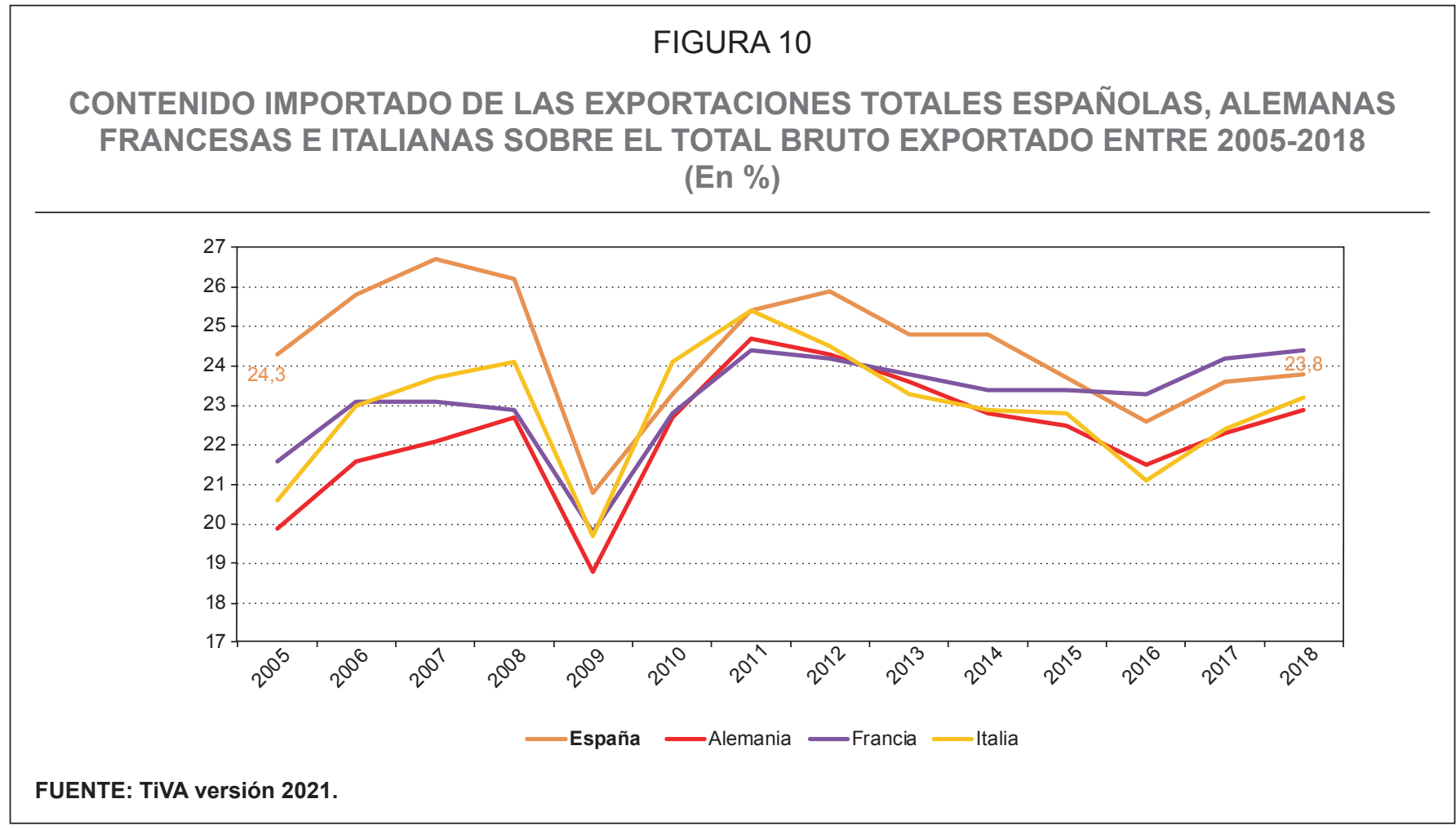

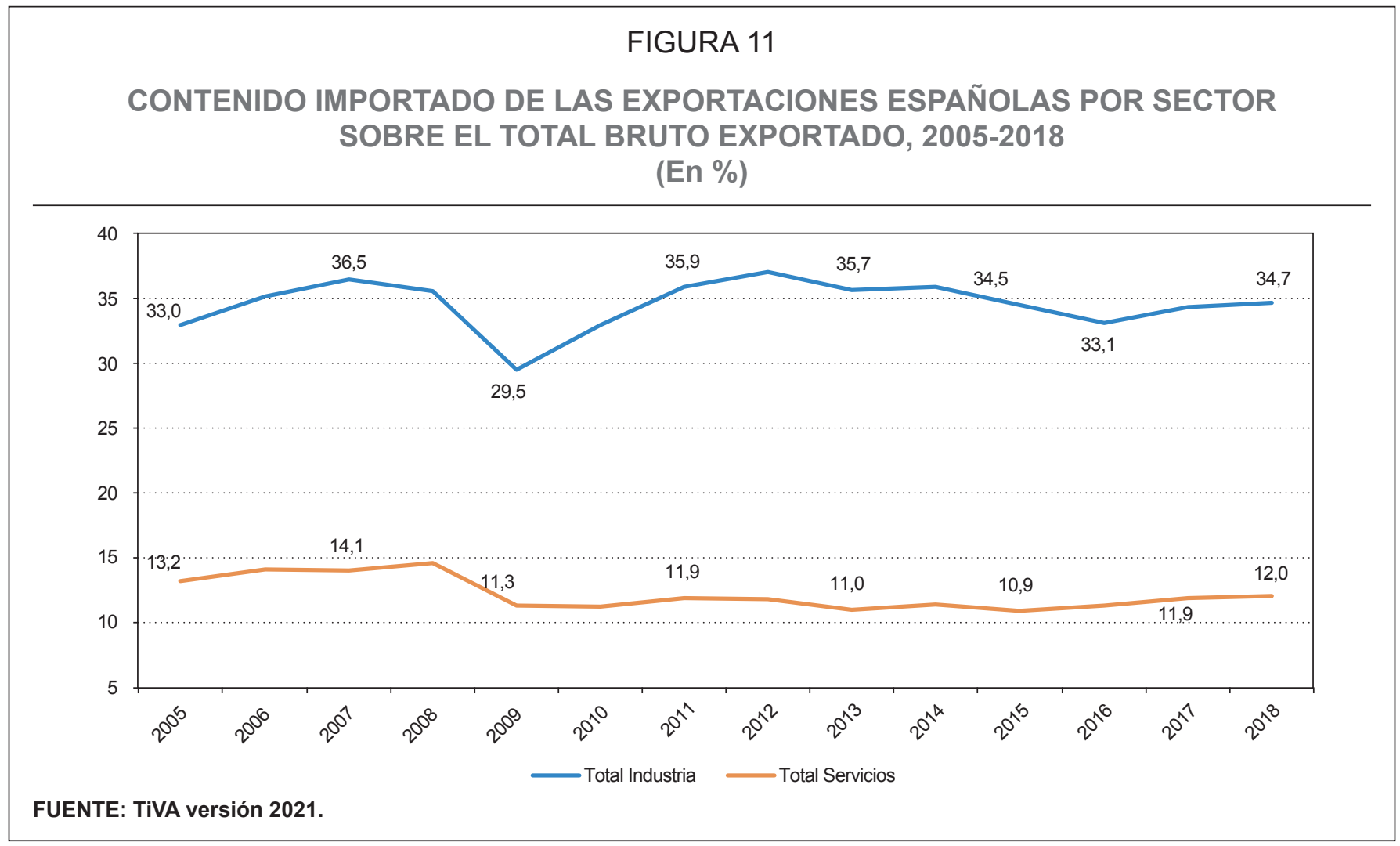


FIGURA 12

\section{VALOR AÑADIDO DOMÉSTICO EN LAS EXPORTACIONES DE AUTOMÓVILES SOBRE EL TOTAL DE EXPORTACIONES DE DICHO SECTOR EN ESPAÑA, FRANCIA, ALEMANIA E ITALIA ENTRE 2005-2018}

(En \%)

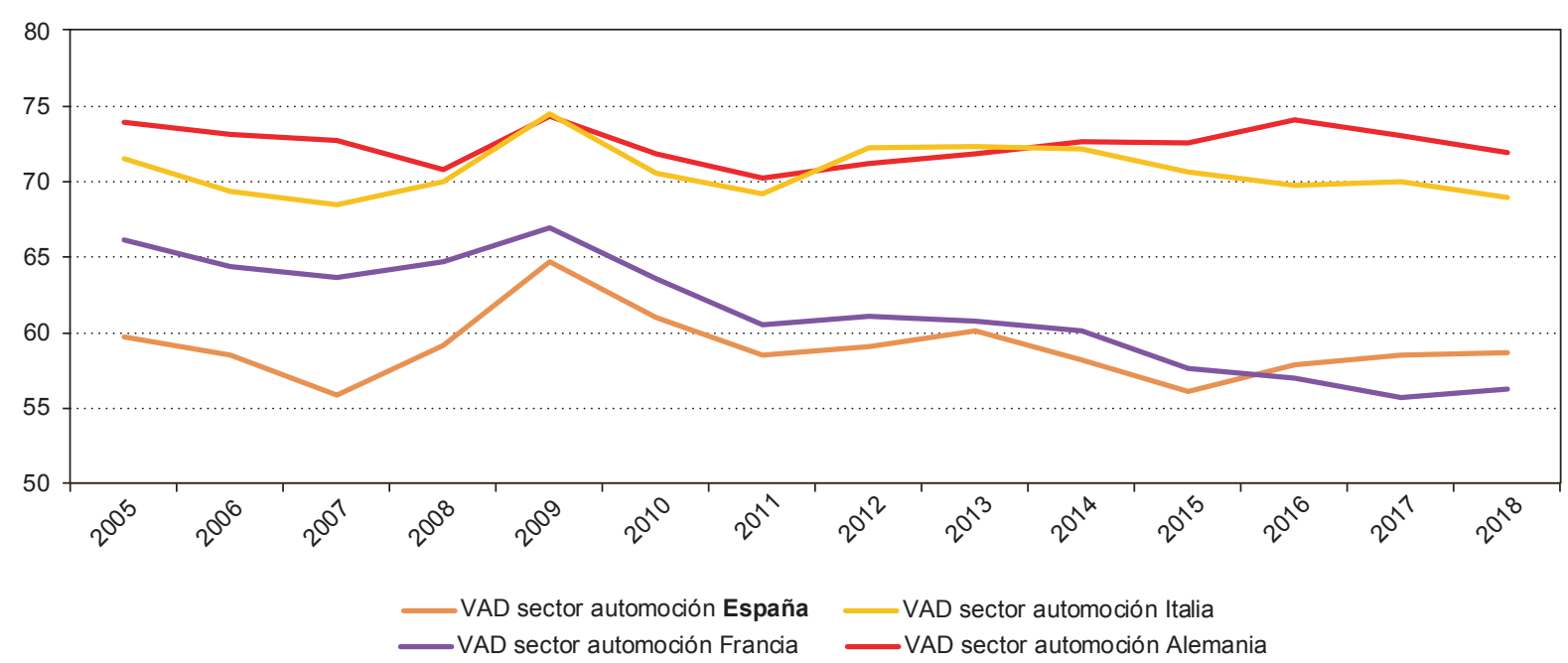

FUENTE: TiVA versión 2021

Francia, si bien continuamos claramente por debajo de Italia y Alemania (Figura 12).

\section{Evolución del valor doméstico incorporado en las importaciones brutas $y$ finales por regiones}

En primer lugar, hay que hacer una distinción entre las importaciones totales que incluyen bienes y servicios intermedios que son reexportados y las importaciones finales que no incluyen los consumos intermedios que son reexportados (demanda final de importaciones). En cuanto al VAD incorporado en las importaciones totales brutas, incluyendo inputs intermedios, España se sitúa en 2018 por encima de 2005 (1 \% en 2018 vs. $0,9 \%$ en 2005) mientras que Alemania ha incrementado su presencia en VAD en sus importaciones (3,4 \%). En Francia y en Reino Unido caen, aunque están mejor que España (-1,8\%). Italia también empeora en el periodo y está en los niveles de España (1\%). En conclusión, hemos mejorado levemente nuestra situación relativa con Francia e Italia, pero no con Alemania.

Pero en realidad lo relevante es ver cómo se comporta el valor añadido doméstico en las importaciones finales y en las exportaciones finales. Veremos ahora cómo en estos dos conceptos España ha mejorado y, sobre todo, cómo ha mejorado frente al resto de sus socios.

\section{Análisis regional comparativo de valor doméstico incorporado en nuestras importaciones finales $y$ en nuestras exportaciones finales}

En este subapartado se describe la intensidad por regiones del VAF contenido en nuestras importaciones entre 2005 y 2018; la intensidad del VAD de España en 
sus exportaciones finales por regiones, y qué importancia tienen los valores añadidos en relación con la demanda final de cada región. En este análisis regional vamos a explorar cómo ha cambiado el comercio o, mejor dicho, el valor del comercio de España, con los tres grandes países de la zona euro, China y resto del mundo. Para ello disponemos del periodo comprendido entre 2005 y 2018 , ambos inclusive. De manera generalizada para España, se ha producido una caída en la proporción de valor añadido foráneo sobre el total de las importaciones finales ${ }^{3}$ procedentes de Europa y del resto del mundo, salvo de China (Figura 13). Esta misma tendencia se da cuando analizamos los valores de VAD español aportado a nuestras exportaciones finales (valor foráneo de importaciones para nuestros socios).

Es decir, que mejora la relación de valor añadido domestico sobre importaciones finales globales y las procedentes de todas las regiones menos China; esto implica menores contribuciones negativas de las importaciones finales al crecimiento económico que en el pasado. Un $73 \%$ en 2018 para el total mundial, frente a un $79 \%$ en 2005.

En lo que respecta a nuestro VAD en las exportaciones finales (Figura 14) 4 , que no de bienes intermedios, se observa un leve incremento con respecto a 2005 del VAD español en la demanda final mundial de $+0,6 \%$ (74,7 \% en 2005 vs. $75,3 \%$ en 2018 ), que es consecuencia de una mejora en las relaciones con China y una caída con respecto a la zona euro - que pasan de $68 \%$ a $66 \%$ - caída que es inferior a la que vimos en la Figura 13 del VAD de la zona euro en sus exportaciones a España, que disminuía de $71 \%$ a $65 \%$. Dada las mejoras en el global mundial de nuestra aportación de valor a nuestras exportaciones y la caída mucho más fuerte del valor foráneo en nuestras importaciones, es de esperar que cualquier recuperación global pos-COVID-19 incorpore más creación de valor del

\footnotetext{
${ }^{3}$ Importaciones finales excluye la importación de consumos intermedios que son reexportados.

${ }^{4}$ Las exportaciones finales excluyen las exportaciones de bienes intermedios que son reexportados.
}

sector exterior que en recuperaciones anteriores. Esta conclusión está en línea con el cambio observado en la relación o elasticidad entre las exportaciones brutas e importaciones brutas antes y después de la pandemia.

Si comparamos ambos conceptos es decir el VAD en nuestras exportaciones y el VAF en nuestras importaciones el resultante es una mejora clara de forma que no situamos ya muy en línea con la zona euro, y con mejor relación de VAD cuando comparamos importaciones y exportaciones finales globales.

\section{Las cadenas de valor y los inputs intermedios}

¿Qué podemos decir de la integración, regional o no, en las cadenas de valor de la economía española durante este periodo en relación con nuestros socios? Una manera de mirarlo es construir a partir de datos TiVA la relación de input intermedios importados sobre el total de importaciones de bienes industriales. Y, posteriormente, analizar la proporción de inputs importados que se utilizan en la exportación manufacturera ${ }^{5}$, es decir, que son reexportados (Figura 15).

Cuando analizamos la proporción de inputs intermedios, tanto bienes como servicios, que son utilizados para generar una actividad económica con el fin de satisfacer la demanda externa, es decir, para exportar, del valor total de las importaciones de bienes y servicios intermedios de España en 2018, el $46,6 \%$ fueron empleadas en las exportaciones de manufacturas, siendo en 2005 del 34,5\% (aunque este aumento no se debe al sector bienes de equipo, como ya se mencionó anteriormente). España es en proporción el país que más creció en esta ratio, y que nos coloca en niveles solo por debajo de Alemania y equiparado a Italia, cuando antes estábamos incluso por detrás de Francia. Tanto en España - como en Alemania en menor medida- la actividad económica que más inputs importados dedica a su exportación es

\footnotetext{
${ }^{5}$ Se podría calcular sobre el total de bienes y servicios, las conclusiones son similares.
} 

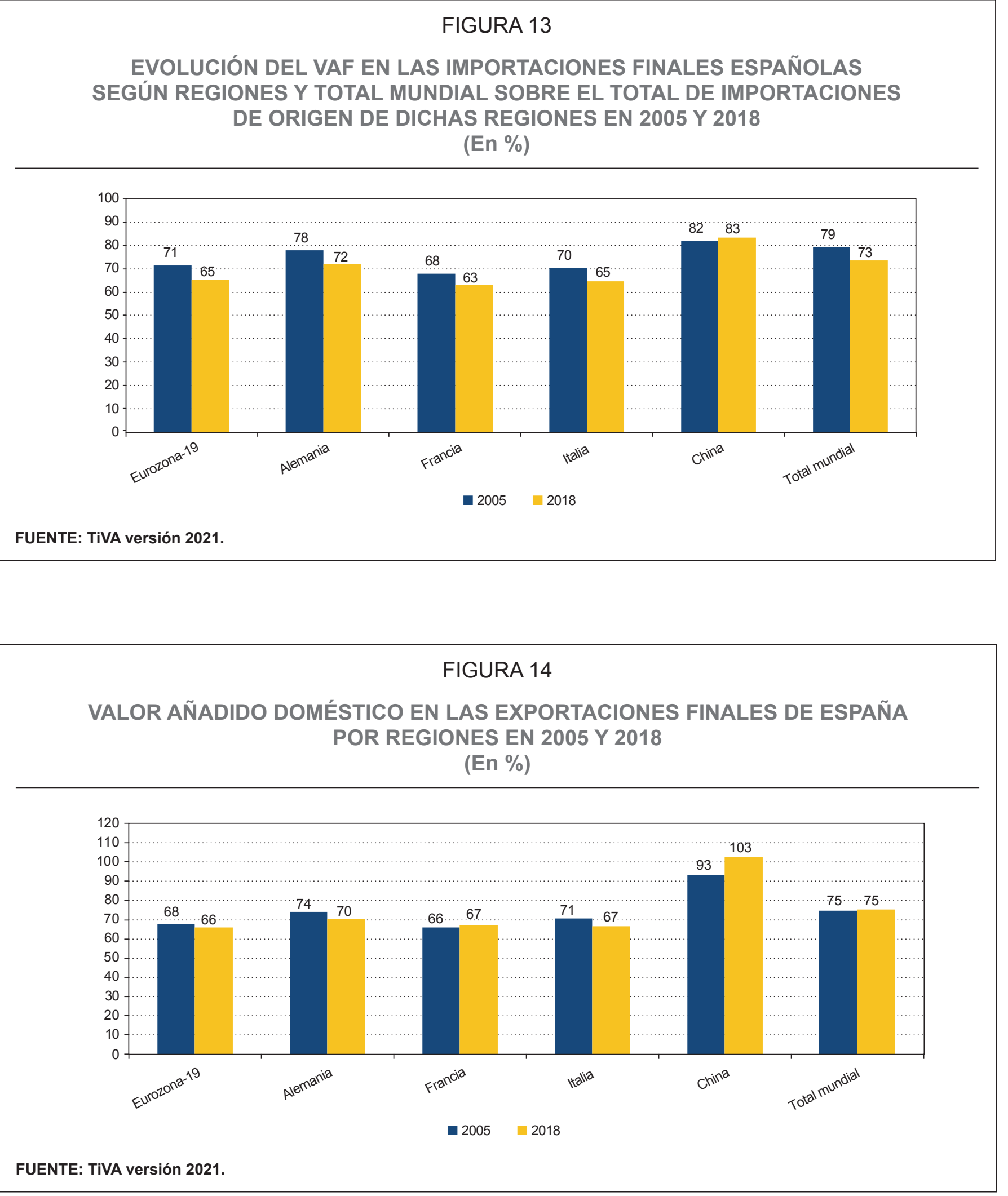


\section{FIGURA 15}

\section{INPUTS IMPORTADOS UTILIZADOS PARA LA EXPORTACIÓN MANUFACTURERA SOBRE EL TOTAL DE INPUTS IMPORTADOS, 2005-2018}

(En \%)

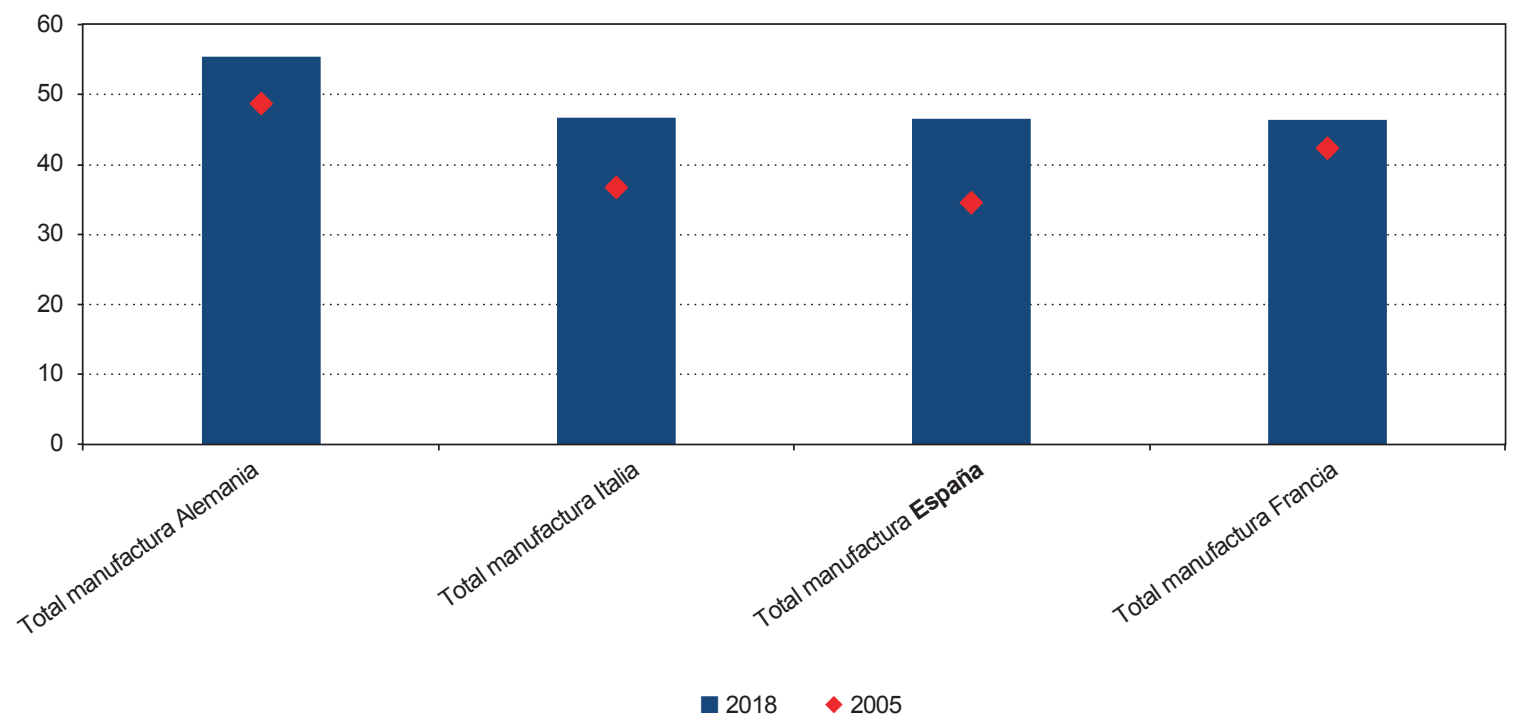

FUENTE: TiVA versión 2021.

«vehículos de motor», y ha experimentado un incremento con respecto a 2005 , destinando las empresas de este sector más del $70 \%$ de inputs importados a satisfacer la demanda externa. En Francia el sector exportador más intensivo en inputs intermedios es "otro equipamiento de transporte», y en Italia, "maquinaria», de ahí el importante peso del valor añadido foráneo en las exportaciones de bienes de equipo italianas.

En conclusión, mejoramos no solo en la relación VAD incorporado en nuestras importaciones finales (subapartado anterior) sino que de las importaciones de insumos intermedios dedicamos cada vez más a la exportación (no así para bienes de equipo) y además los inputs intermedios pesan más en nuestras importaciones, porque estamos más presentes en cadenas de valor. Esto es por lo tanto un buen dato para la creación de valor del sector exterior.

\section{Evolución del contenido de servicios (domésticos y foráneos) en las EBBSS}

A diferencia de lo ocurrido en Italia y Alemania, en España y Francia la influencia de los servicios en la economía industrial-manufacturera se ha incrementado respecto a 2005, es decir, respecto al periodo precrisis, desde el $35 \%$ al $36 \%$ en España y desde el $38,1 \%$ al $39,2 \%$ en Francia.

No obstante, analizando más detenidamente los cambios en las características del comercio, en España, el incremento de la presencia de los servicios en las exportaciones brutas ha sido por servicios españoles o domésticos, mientras que el valor añadido foráneo de los servicios incorporados en las exportaciones ha caído con respecto a 2005.

En esta variable, España mejora frente a los otros tres grandes de la zona euro, señalando que el valor 
doméstico en servicios de las exportaciones brutas ha mejorado significativamente comparado con Alemania, Italia y Francia. Así, del total del $59 \%$ de servicios incorporados, el $49 \%$ eran domésticos, frente al $45 \%$ en 2005 (Figura 16). Es decir, la servicificación en este periodo en España se ha hecho con VAD, lo cual es muy positivo.

En cuanto a solo manufacturas, en España el VA de servicios contribuyó en 2018 un $36 \%$ al valor de las exportaciones manufactureras, con la mayor proporción en los sectores alimenticio (40 \%) y madera, papel e impresión (35,6 \%). Esto refleja la gran importancia relativa de los servicios en nuestra economía manufacturera, similar al italiano y al francés y superior al alemán (33,4 \%). Mirándolo desde otro punto de vista, las exportaciones de manufacturas alemanas son realmente más manufactureras, y no solamente más altas.

Lo relevante es que en este proceso de servicificación, el sector que más ha disminuido la presencia de servicios foráneos en sus exportaciones en España es TIC y electrónica, y el que más ha incrementado la presencia doméstica ha sido el de minería. Con ello se aprecia una reorientación productiva y un esfuerzo por mejorar la calidad y el valor de lo producido domésticamente, siendo menos dependiente de los servicios provenientes del exterior las exportaciones que incorporan más tecnología. Por lo tanto, es una tendencia claramente positiva y que señala también la mejora en cuanto a calidad y tecnología de nuestras exportaciones. También indicaría que no es tanto el número de empresas exportadoras lo que cuenta como el valor añadido de las exportaciones vía aumento de servicios domésticos incorporados a las mismas.

\section{Recuperación y comercio internacional.}

Algunas consideraciones sobre el comercio global pos-COVID-19 y el crecimiento económico

La pandemia de COVID-19 provocó en la primera mitad de 2020 una de las mayores reducciones en el comercio y los volúmenes de producción desde la Segunda Guerra Mundial.

Tras este primer colapso inicial, el comercio de bienes tuvo una recuperación más rápida y vigorosa de lo esperado. De hecho, apenas seis meses después de tocar suelo, en noviembre de 2020 el comercio de bienes ya había vuelto a los niveles prepandemia alentado por los efectos combinados de una liberación de la demanda reprimida y rotación en la demanda hacia algunos bienes duraderos, que tienen una alta intensidad comercial. En contraposición, tras la crisis financiera global (GFC) se necesitaron 26 meses para alcanzar niveles precrisis; la disminución generalizada de la demanda se vio exacerbada por la sequía de la financiación del comercio.

Por el contrario, durante la crisis del COVID-19 el comercio de servicios disminuyó más del doble que el comercio de bienes. Además, los datos agregados, tanto bienes como servicios, enmascaran una enorme heterogeneidad, tanto en la magnitud del declive como de la recuperación, por productos (Arriola et al., 2021). Esto se explica por el hecho de que la pandemia y las respuestas políticas asociadas han inducido grandes cambios en la demanda (por cambio de hábitos) y oferta del comercio internacional, por diferentes costes de ajuste entre productos y países, y problemas de suministro de componentes.

Los impactos en la crisis del COVID-19 en la distribución del comercio entre regiones y países también han sido grandes. Por el lado de la oferta, en un primer momento China y otros países asiáticos fueron los más exitosos en llenar las brechas de suministro resultantes de los bloqueos y cambios en la demanda en otras regiones. Por el lado de la demanda, las importaciones de los países desarrollados, con EE UU a la cabeza, fueron las primeras en recuperar los niveles previos. Esto se debe a la fuerte magnitud de los estímulos públicos aprobados en los países desarrollados.

Algunos de estos desarrollos comerciales serán de corta duración, pero la pandemia y la respuesta de políticas también provocarán cambios permanentes en el 


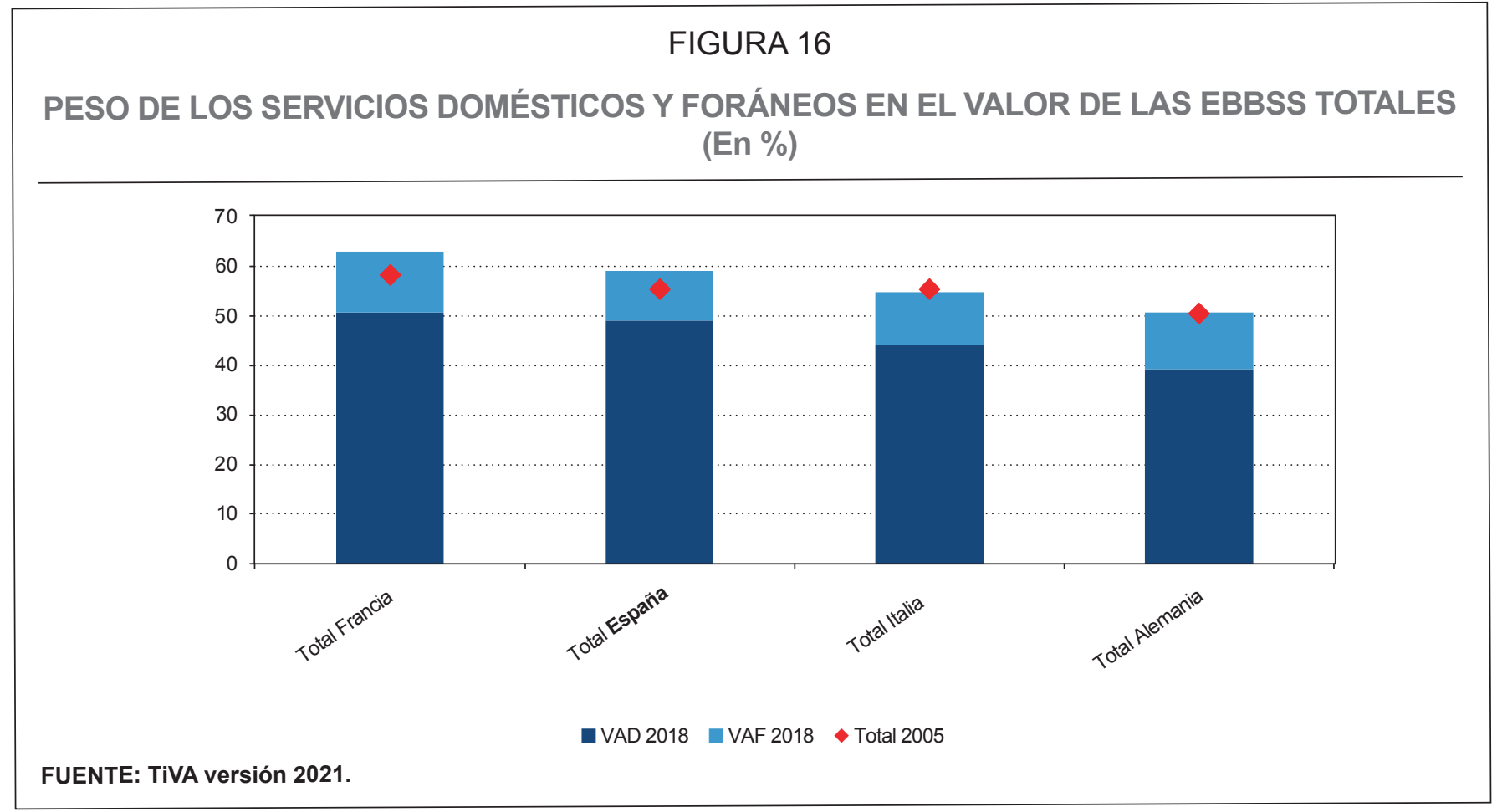

comercio. A continuación, nos preguntamos cuáles pueden ser algunos de estos cambios a más largo plazo.

\section{Posibles implicaciones a más largo plazo de esta crisis para el comercio}

En primer lugar, por el lado de la oferta, en la medida que esta pandemia ha revelado cuellos de botella y vulnerabilidades no detectadas hasta ahora, es probable que algunas empresas se dediquen a adoptar nuevas estrategias de mitigación de riesgos buscando aumentar la resiliencia y mitigar problemas logísticos. Estas estrategias podrían llevar a las empresas a querer mantener inventarios más grandes, y diversificar proveedores y sitios de producción (Saurav et al., 2020). La escasez de suministro de bienes esenciales y componentes críticos al inicio de la pandemia atrajo la atención de los gobiernos, que pueden presionar para una mayor autosuficiencia de ciertos productos críticos. Pero más allá de eso, no cabe esperar una gran reconfiguración de las GVC. Tanto algunos estudios académicos como la evidencia empírica de esta crisis apuntan a que las empresas con una producción más integrada en las GVC, aunque están más expuestas a perturbaciones de oferta generadas en los países en los que se encuentran sus proveedores, también tienden a resistir mejor las crisis y tienen una mayor capacidad para recuperarse (Arriola et al., 2021).

En segundo lugar, la pandemia inducirá importantes cambios en la demanda, por la reconfiguración de hábitos, o el aumento del teletrabajo o de las compras en línea. También la respuesta a la pandemia afectará de forma diferente al crecimiento de nuestros socios comerciales. Mientras que el inicio de la pandemia desencadenó la recesión mundial más sincronizada de la historia, la recuperación posterior y perspectivas de cicatrices a largo plazo se está caracterizando por una gran divergencia, dependiendo del margen de maniobra para prolongar los estímulos y la composición sectorial preexistente. 
Mientras que en la crisis financiera global fueron los países desarrollados los que sufrieron las mayores cicatrices económicas, en esta crisis parece que serán los países emergentes los que sufrirán más. Según el FMI, en 2024, las economías occidentales tendrán un nivel de PIB similar al que se proyectaba antes de la crisis. Sin embargo, las economías emergentes presentarán un PIB un 6,2 \% inferior, y un $8 \%$ inferior si excluyésemos a China. Así pues, la rotación en la economía global hacia un mayor peso de los emergentes puede frenarse tras la crisis del COVID-19.

Esto en el caso de España supondría una mayor importancia del comercio intrazona euro donde hemos visto que ha mejorado mucho la capacidad de generar más valor que nuestros socios a igual crecimiento económico, tanto por la mejora en nuestras elasticidades de exportación respecto del PIB europeo, como por la caída de la elasticidad de importación respecto a nuestro PIB. A esto se le une la mejora en el mayor valor añadido por importación final y la mayor utilización de insumos intermedios para exportaciones de manufacturas.

Existen otros potenciales cambios no ligados a la pandemia. Así el comercio bilateral de los países europeos con los países emergentes podría sufrir más que el comercio intraeuropeo o con otros países desarrollados también por la idea de establecer aranceles basados en las emisiones de carbono para las importaciones procedentes de países que no adopten medidas para reducir sus emisiones de gases de efecto invernadero.

Además de la rotación en la composición del comercio por países, también podría darse una rotación en la composición de la demanda dentro de cada país. En principio, gracias a las generosas ayudas recibidas, los hogares de los países desarrollados, con EE UU a la cabeza, están armados con un arsenal de ahorros excedentes. Esto debería hacer que, a corto plazo, el repunte del gasto de los consumidores en los países desarrollados sea el principal motor del crecimiento del comercio. Pero la inversión, un componente muy castigado tras la anterior crisis financiera, y que es más intensivo en comercio, ahora se está comportando más favorablemente.
Y a más largo plazo, se han está creando incentivos para que las empresas realicen gastos de capital adicionales; las limitaciones de la oferta de mano de obra incitan a las empresas a invertir más en tecnologías que ahorran mano de obra, y las interrupciones logísticas a invertir en la resiliencia de la cadena de suministro. Todo ello se une a la necesidad de avanzar hacia una inversión más verde ante las presiones existentes del imperativo de la sostenibilidad. Y gracias a la intervención de los bancos centrales las condiciones de financiación se han vuelto extremadamente acomodaticias. Es verdad que los desincentivos a inversiones consideradas no sostenibles pueden originar menos inversión y sobre todo más inflación.

Todos estos cambios apuntan a que la «nueva normalidad» del comercio mundial tras el COVID-19 no sea volver a la dinámica del comercio boyante en la década de los años noventa y principios de la de dos mil, que puede haber sido lo excepcional (reducción de barreras comerciales, difusión de nuevas tecnologías, y apertura de la economía china contribuyendo a una caída en el precio relativo de los bienes transables), pero sí puede retomar cierta aceleración respecto a los niveles de los últimos años.

Estas perspectivas de crecimiento de las importaciones en países que representan más del $70 \%$ de nuestras exportaciones también jugarían a favor del sector exterior español.

Por otra parte, la evolución del comercio parece que estará determinada cada vez menos por los bienes que por los servicios. Ya antes del COVID-19, el comercio de servicios no sufría una desaceleración como sí la estaba sufriendo el comercio de bienes, y en el periodo posterior a la GFC el comercio de servicios siguió creciendo más rápido que el PIB. Y tras la pandemia, la adopción más acelerada y generalizada de algunas tecnologías digitales podría abrir la puerta a un mayor comercio de servicios.

En general, las trabas al comercio en servicios y sus costes siguen siendo mayores que las del comercio de bienes (Benz, 2017), por lo que para capitalizar la posibilidad de crecer en las exportaciones de servicios 
será clave seguir avanzando en armonizar las regulaciones de estos sectores. Además, dado que los servicios negociables son vínculos clave entre las etapas de las cadenas de valor y facilitadores del comercio de bienes, el mayor desarrollo de servicios de comunicaciones, finanzas o logística ayudaría no solo a estimular las exportaciones de servicios, sino también las de bienes.

\section{La crisis del COVID-19 en España y sus diferencias con la Unión Europea}

Realmente lo relevante o diferencial de esta crisis del COVID-19 es que es una crisis no provocada por desequilibrios financieros o económicos. Pese a su profundidad, podemos ya decir que la duración de esta crisis va a ser más limitada en el tiempo que la GFC, aunque no sabemos cómo serán las cicatrices en algunos sectores. También sabemos que la velocidad de recuperación será desigual ya que las políticas de confinamiento y la importancia relativa de los sectores económicos ligados a la movilidad son distintas por países. Para ver la potencial contribución del sector exterior tenemos que ver los factores diferenciales españoles respecto a nuestros principales socios y que se podrían resumir en el mayor peso del sector servicios turístico en nuestro país, baste ver los datos de la importancia de las exportaciones de turismo (Figura 17).

En 2020, el PIB español sufrió un abrupto retroceso del $-10,8 \%$ anual medio, muy superior al descenso del PIB en el conjunto de la zona euro del $-6,6 \%$, básicamente por el mayor peso del sector servicios turísticos y por la severidad del confinamiento. Una de las características de esta crisis es que el desplome de las rentas reales ha sido claramente inferior a otras y a la caída del consumo privado y que la respuesta del consumo público ha sido mayor. Ahora bien, si comparamos la zona euro con España podemos ver determinadas diferencias (Figura 18).

En primer lugar, la caída de la renta disponible de los hogares españoles fue mucho mayor y todavía no hemos recuperado los niveles precrisis, mientras en la zona euro los recuperaron en el tercer trimestre de 2020. Es decir, tendrían más potencial de crecimiento del consumo en los próximos trimestres que España y, además, dada nuestra menor elasticidad de las importaciones al consumo, debería producirse una contribución adicional del sector exterior. Es muy llamativo que los niveles de consumo privado tengan una evolución tan pareja entre España y la zona euro, frente a la evolución dispar de las rentas de los hogares que en el caso español no deja de caer desde el tercer trimestre de 2020.

Si miramos el PIB por el lado de la oferta vemos que los dos sectores privados que están peor en relación con su nivel previo en España son el turismo y la industria, mientras que en la zona euro la industria ya recuperó los niveles precrisis. Mientras, un sector como el de las actividades relacionadas con el sector público (AA PP, sanidad y educación) está claramente contribuyendo más al PIB en España que en la zona euro y en ambos casos por encima del nivel de 2019 (el crecimiento bianual del empleo — comparado con 2019_ es del $7 \%$ en España).

En resumen, la potencial recuperación del consumo en Europa deberá ser más fuerte y rápida que en España dado que no ha caído la renta de los hogares y en cualquier caso España tiene una mayor elasticidad de exportaciones de servicios al PIB europeo y deberíamos ver una clara mejoría, en el corto plazo. Por último, si con los fondos comunitarios la inversión en bienes de equipo aumenta mucho más que en nuestros socios, habrá que ver si se mantiene la ventaja en el sentido de una menor intensidad de importaciones por bienes de equipo en España.

\section{Conclusiones}

Con todas las precauciones, y siempre dependiendo de cómo evolucione el PIB mundial y por regiones, podemos ser optimistas sobre el papel del sector exterior español en esta fase de recuperación y crecimiento. Por una 


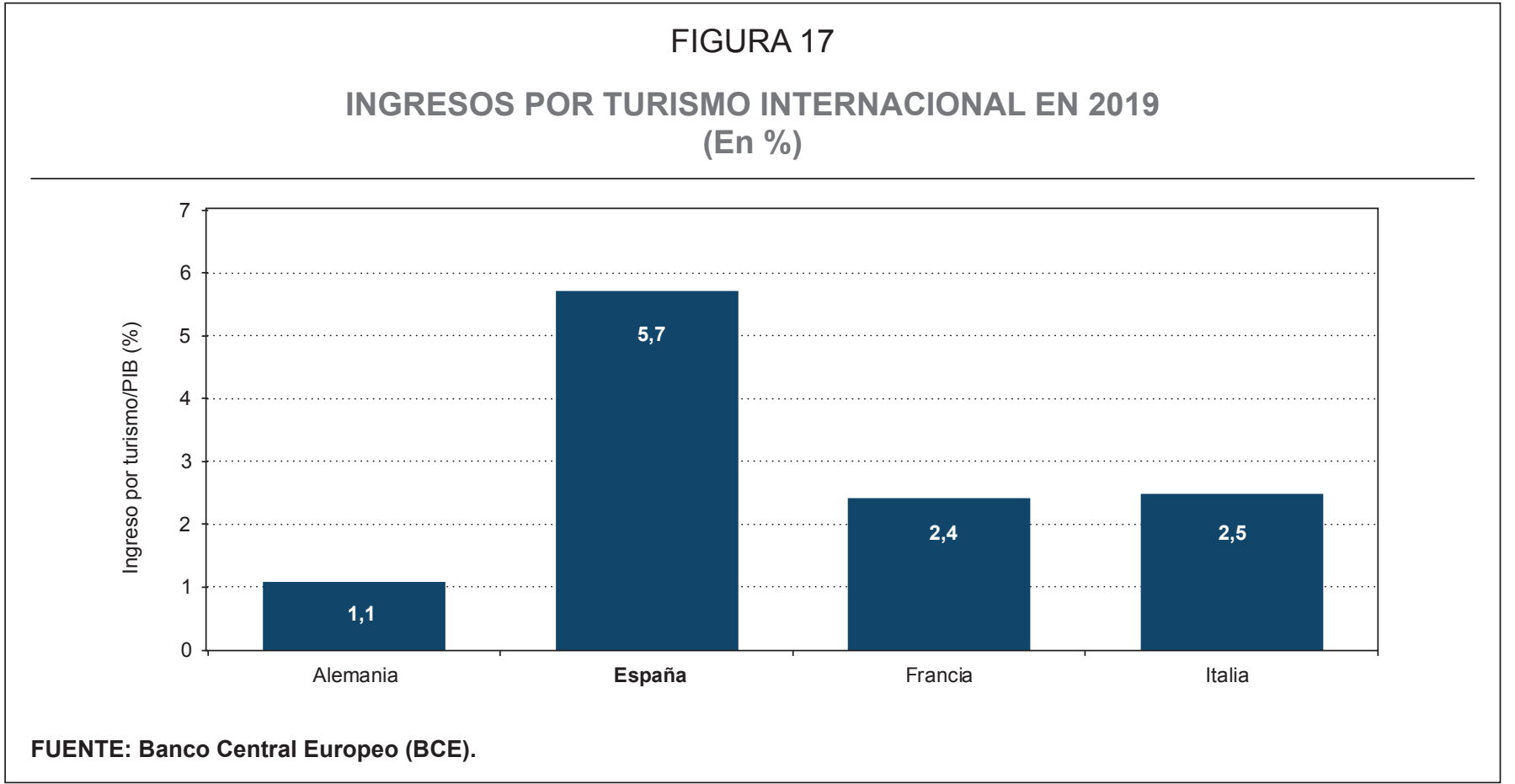

\section{FIGURA 18}

CONSUMO Y RENTA BRUTA DISPONIBLE EN LA ZONA EURO Y EN ESPAÑA (NIVELES)

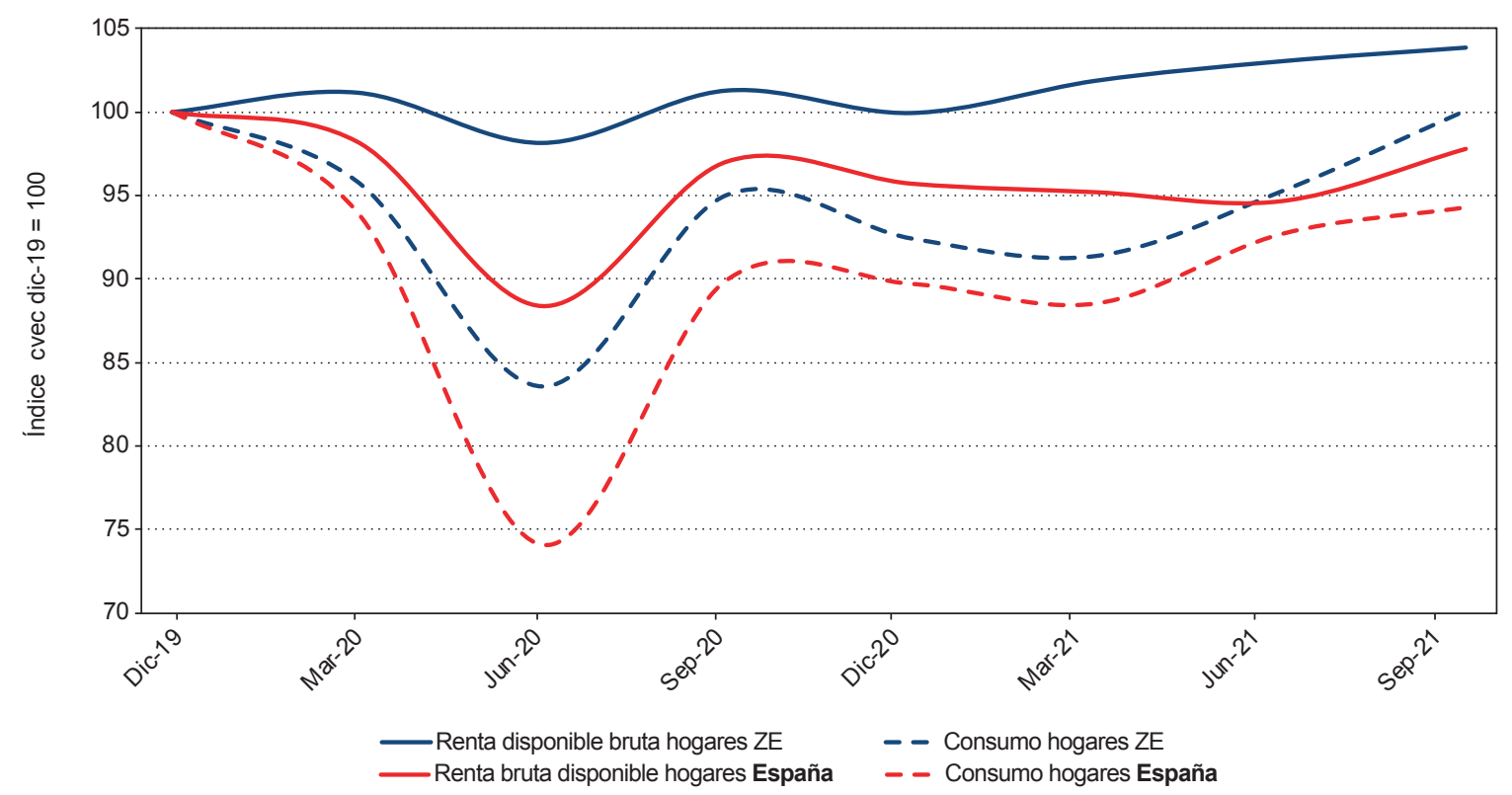

FUENTE: Eurostat e INE. 
parte, el previsible aumento de la relación comercio exterior y PIB, especialmente entre países desarrollados, nos favorece dada nuestra menor elasticidad de importaciones frente a la UE y que incorporamos menor valor foráneo que ellos en nuestras respectivas importaciones. La evolución en el periodo 2014-2019 y las tendencias subyacentes del sector exterior español hacia un comportamiento similar, y en muchas variables mejor que el de la zona euro en términos de valor añadido en las exportaciones y también mejor en cuanto a la proporción de inputs reexportados, también es una señal muy positiva a tener en cuenta. La no pérdida de competitividad del factor trabajo durante la anterior etapa de crecimiento, tras las ganancias del periodo de crisis anterior; el mejor track record en reformas de mercados de productos y de factores son también datos positivos. El aumento de inputs importados que son reexportados, indica una mayor participación en las cadenas de valor, lo cual también es positivo. La creciente servificación de nuestras exportaciones está teniendo lugar con una mayor participación y crecimiento de servicios domésticos frente a foráneos; la concentración de esta servificación en sectores de media y alta tecnología y en otros donde la mejora de la calidad de productos es clave para exportar más -léase, por ejemplo, el sector de alimentación- son todos factores que indican una mejora del posicionamiento de nuestro sector exterior. Por lo tanto, tras las recuperaciones esperadas en la movilidad en 2022 y en el turismo, todo indica que el sector exterior contribuirá positivamente al crecimiento. Por supuesto que la política regulatoria, fiscal y de cambio climático en los dos últimos años, que no son consecuencia de la pandemia, pueden traducirse en una pérdida de competitividad que dañe nuestro crecimiento. Entender esta restricción y continuar con las mejoras es clave no solo para permitir una contribución mayor del sector exterior, sino para aumentar la duración e intensidad de la recuperación que es de lo que se trata.

En resumen si se profundiza en las tendencias de mejora vistas en el periodo 2014-2019; si no se retrocede en reformas de mercados de factores; si se produce un esperado aumento del comercio internacional, especialmente en países OCDE, y si la mejora de las vacunaciones en Europa se traduce en una mejora rápida de nuestras exportaciones de servicios turísticos, el sector exterior español continuará contribuyendo al crecimiento económico de manera clave en esta recuperación y en los posteriores años de crecimiento.

\section{Referencias bibliográficas}

Altomonte, C., Di Mauro, F., Ottaviano, G., Rungi, A. \& Vicard, V. (2012). Global Value Chains during the Great Trade Collapse: A Bullwhip Effect? ECB, Working Paper No. 1412. January.

Antràs, P. (2020). Conceptual Aspects of Global Value Chains. World Bank Group, Policy Research Working Paper No. 9114. Washington, DC.

Arriola, C., Kowalski, P. \& van Tongeren, F. (2021). The impact of COVID-19 on directions and structure of international trade. OECD, Trade Policy Papers No. 252. OECD Publishing, Paris.

Baldwin, R. (2011). 21 ${ }^{\text {st }}$ Century Regionalism: Filling the gap between $21^{\text {st }}$ century trade and $20^{\text {th }}$ century trade rules. WTO, Staff Working Paper ERSD-2011-08.

Banco de España. Informes de balanza de pagos y posición de inversión internacional. https://www.bde.es/webbde/ es/estadis/infoest/temas/sb_extbppii.html

Benz, S. (2017). Services trade costs: Tariff equivalents of services trade restrictions using gravity estimation. OECD, Trade Policy Papers No. 200. OECD Publishing, Paris.

Boz, E., Bussière, M. \& Marsilli, C. (2014). Recent slowdown in global trade: Cyclical or structural. VoxEU CEPR Policy. November 12.

Casella, B., Bolwijn, R., Moran, D. \& Kanemoto, K. (2019). Improving the analysis of global value chains: the UNCTAD-Eora Database. UNCTAD Transnational Corporations, 26(3), 115-142.

Constantinescu, C., Mattoo, A. \& Ruta, M. (2015). The Global Trade Slowdown: Cyclical or Structural? IMF, Working Paper No. 6.

Fernald, J. \& Li, H. (2021). The Impact of COVID on Potential Output. Federal Reserve Bank of San Francisco, Working Paper Series No. 9.

Freund, C. (2009). The Trade Response to Global Downturns: Historical Evidence. World Bank, Policy Research Working Paper No. 5015.

Haugh, D., Kopoin, A., Rusticelli, E., Turner, D. \& Dutu, R. (2016). Cardiac Arrest or Dizzy Spell: Why is World Trade So Weak and What can Policy Do About It? OECD, Economic Policy Papers No. 18. OECD Publishing, Paris. 
Hoekman, B. (Ed.). (2015). The Global Trade Slowdown. A New Normal? VoxEU.org eBook, CEPR Press and EUI.

Koopman, R., Wang, Z. \& Wei, S. J. (2014). Tracing valueadded and double counting in gross exports. American Economic Review, 104(2), 459-494.

Lanz, R. \& Maurer, A. (2015). Services and Global Value Chains - Some evidence on Servicification of Manufacturing and Services Networks. WTO, Working Paper ERSD-2015-03.

Lipsey, R. E. (2009). Measuring International Trade in Services. In M. Reinsdorf \& M. J. Slaughter (Eds.), International Trade in Services and Intangibles in the Era of Globalization (pp. 27-70). University of Chicago Press.

Marschinski, R. \& Martínez-Turégano, D. (2020). The EU's shrinking share in global manufacturing: a value chain decomposition analysis. National Institute Economic Review, 252, R19-R32.

MINCOTUR, Ministerio de Industria, Comercio y Turismo. Informes de Comercio Exterior. https://comercio.gob. es/ImportacionExportacion/Informes_Estadisticas/ Paginas/Informes-periodicos.aspx
Miroudot, S. (2019). Services and Manufacturing in Global Value Chains: Is the Distinction Obsolete? $A D B I$, Working Papers Series No. 927. https://ideas.repec.org/p/ris/ adbiwp/0927.html

Razin, A. (2021). Globalization, Migration, and Welfare State: Understanding the Macroeconomic Trifecta. PalgraveMacmillan.

Rodrik, D. (2011). The Globalization Paradox: Democracy and the Future of the World Economy. W. W. Norton \& Company.

Saurav, A., Kusek, P., Kuo, R. \& Viney, B. (2020). The Impact of COVID-19 on Foreign Investors: Evidence from the Quarterly Global MNE Pulse Survey for the Third Quarter of 2020. World Bank Group.

Timmer, M. P., Los, B., Stehrer, R. \& de Vries, G. J. (2013). Fragmentation, incomes and jobs: an analysis of European competitiveness. Economic Policy, 28(76), 613-661.

TiVA, Trade in Value Added Database. (2018). Recuperado de la base de datos de la Organización para la Cooperación y el Desarrollo Económicos (OCDE).

UNCTAD, United Nations Conference on Trade and Development. (2020). World Investment Report 2020: International Production Beyond the Pandemic. 


\section{ANEXO}

\section{RELACIÓN ENTRE EL PIB REAL Y LAS IMPORTACIONES DE BIENES Y SERVICIOS}

En el presente Anexo se ha realizado un análisis sencillo de la relación existente entre el PIB real y las importaciones de bienes y servicios en el periodo comprendido entre el primer trimestre de 2001 y el cuarto trimestre de 2019, se han excluido tanto el periodo que va desde el primer trimestre de 2008 hasta el primer trimestre de 2009 y del primer trimestre de 2020 en adelante, porque son periodos de crisis y pueden alterar la relación a largo plazo que se pretenden identificar.

Realizando la representación de la Figura 1 del Anexo en niveles del PIB y las importaciones de bienes y servicios, se puede observar a simple vista cómo parece que existe una relación a largo plazo entre ambas variables y que, dicha relación, cambia a raíz de la crisis financiera de 2008.

\section{FIGURA 1}

\section{RELACIÓN PIB E IMPORTACIONES DE BIENES Y SERVICIOS}

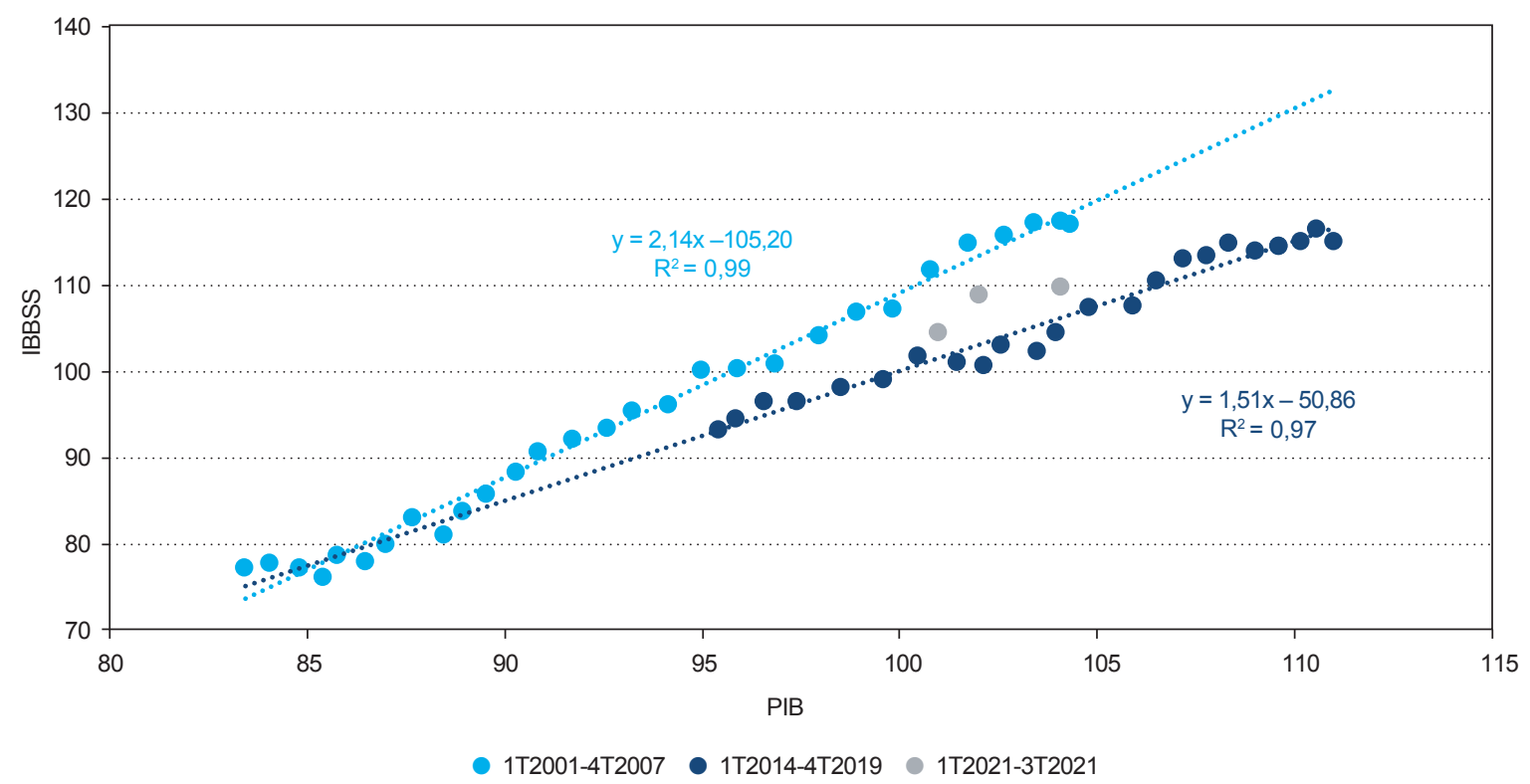

FUENTE: INE.

Para comprobar estadísticamente la existencia de dicha relación y que no se trata de una relación espuria, se utiliza el test de Granger (Tabla 1 del Anexo). Los resultados de dicho test dan que se rechaza la hipótesis nula, es decir, que no podemos descartar que exista causalidad entre ambas variables en ambos sentidos. 
ANEXO (Continuación 1)

RELACIÓN ENTRE EL PIB REAL Y LAS IMPORTACIONES DE BIENES Y SERVICIOS

TABLA 1

TEST DE GRANGER

\begin{tabular}{|c|c|c|c|}
\hline $\begin{array}{l}\text { Pairwise Granger Causality Tests } \\
\text { Date: 12/20/21 Time: 18:48 } \\
\text { Sample: 2001Q1 201904 } \\
\text { Lags: } 2\end{array}$ & & & \\
\hline Null Hypothesis: & Obs & F-Statisoc & Prob. \\
\hline $\begin{array}{l}\text { PIB does not Granger Cause IBBSS } \\
\text { IBBSS does not Granger Cause PIB }\end{array}$ & 69 & $\begin{array}{l}9.65940 \\
5.64289\end{array}$ & $\begin{array}{l}0.0002 \\
0.0055\end{array}$ \\
\hline
\end{tabular}

FUENTE: INE y elaboración propia.

Por otra parte, para confirmar la existencia de un cambio de la relación en el largo plazo, o cambio estructural, se ha realizado una regresión sencilla cuya finalidad es establecer la relación directa entre ambas variables y ver, a través de la utilización de variables dummy, si existe dicho cambio estructural (Tabla 2 del Anexo).

TABLA 2

\section{MODELO IBBSS EN FUNCIÓN DEL PIB ESPAÑA}

\begin{tabular}{|c|c|c|c|c|}
\hline \multicolumn{5}{|c|}{$\begin{array}{l}\text { Dependent Variable: IBBSS } \\
\text { Method: ARMA Maximum Likelihood (OPG-BHHH) } \\
\text { Date: 12/20/21 Time: 19:33 } \\
\text { Sample: 2001Q1 2019Q4 } \\
\text { Included observations: } 71 \\
\text { Convergence achieved after } 106 \text { iterations } \\
\text { Coeffi cient covariance computed using outer product of gradients }\end{array}$} \\
\hline Variable & Coefficient & Std. Error & t-Statistic & Prob. \\
\hline C & -86.69903 & 22.48037 & -3.856654 & 0.0003 \\
\hline PIB & 1.963873 & 0.223454 & 8.788707 & 0.0000 \\
\hline D0209 & -14.26953 & 1.840437 & -7.753337 & 0.0000 \\
\hline $\mathrm{AR}(1)$ & 0.826793 & 0.059452 & 13.90693 & 0.0000 \\
\hline $\mathrm{MA}(3)$ & 0.359172 & 0.153225 & 2.344074 & 0.0221 \\
\hline SIGMASQ & 2.040187 & 0.404706 & 5.041163 & 0.0000 \\
\hline R-squared & 0.984850 & \multicolumn{2}{|c|}{ Mean dependent var } & 97.54836 \\
\hline Adjusted R-squared & 0.983684 & \multicolumn{2}{|c|}{ S.D. dependentvar } & 11.68704 \\
\hline S.E. of regression & 1.492820 & \multicolumn{2}{|c|}{ Akaike info criterion } & 3.771488 \\
\hline Sum squared resid & 144.8533 & \multicolumn{2}{|c|}{ Schwarz criterion } & 3.962700 \\
\hline Log likelihood & -127.8878 & \multicolumn{2}{|c|}{ Hannan-Quinn criter. } & 3.847527 \\
\hline F-statistic & 845.0689 & \multirow{2}{*}{\multicolumn{2}{|c|}{ Durbin-Watson stat }} & 2.208802 \\
\hline Prob(F-statistic) & 0.000000 & & & \\
\hline Inverted AR Roots & .83 & & & \\
\hline Inverted MA Roots & .36-.62í & $.36+.62 \mid$ & .71 & \\
\hline
\end{tabular}

FUENTE: INE y elaboración propia. 


\section{ANEXO (Continuación 2)}

\section{RELACIÓN ENTRE EL PIB REAL Y LAS IMPORTACIONES DE BIENES Y SERVICIOS}

En el modelo se ha introducido la variable dummy D0209, que toma el valor 0 en el primer periodo estudiado y 1 en el segundo. Esta variable dummy es significativa, podemos ver cómo claramente la hipótesis nula de que sea 0 se rechaza, por lo que podemos decir que la relación entre el PIB y las importaciones de bienes y servicios cambia en el segundo periodo analizado.

El análisis de los residuos del modelo muestra que estos se mantienen dentro de los intervalos de confianza, ajustándose el modelo considerablemente bien a la serie original, además de la ausencia de autocorrelación y que siguen una distribución normal (Figura 2, Figura 3 y Tabla 3 del Anexo).

FIGURA 2

\section{MODELO RESIDUOS}

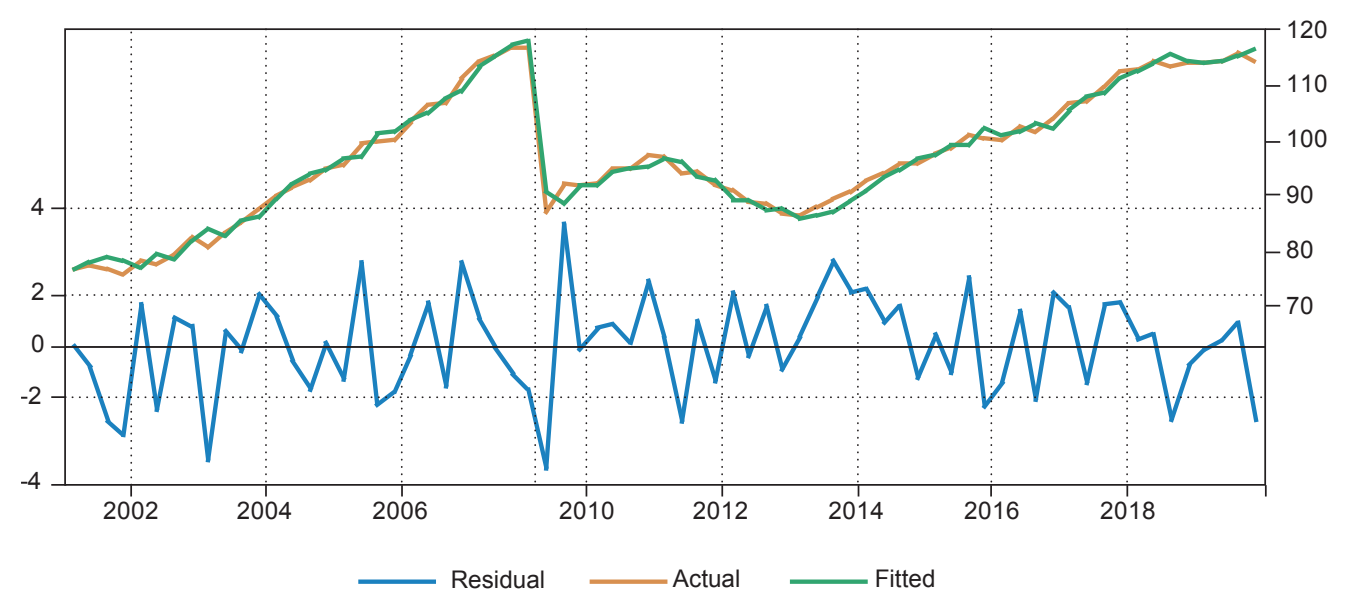

FUENTE: INE y elaboración propia.

FIGURA 3

TEST DE NORMALIDAD DE LOS RESIDUOS

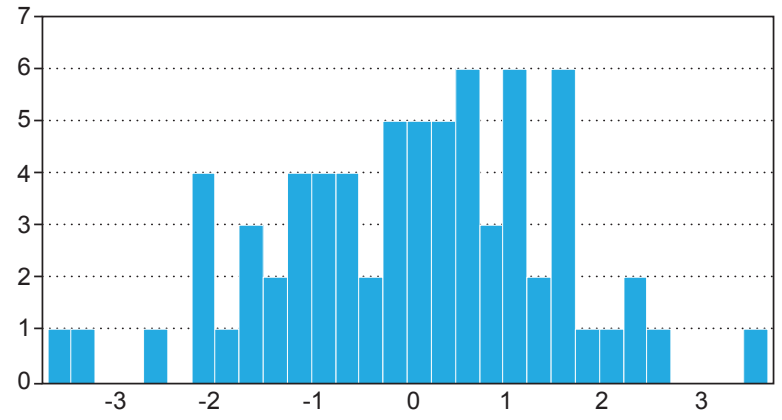

\begin{tabular}{lr}
\multicolumn{2}{l}{ Series: Residuals } \\
\multicolumn{2}{l}{ Sample 2001Q1 2019Q4 } \\
Observations 71 \\
Mean & 0.053833 \\
Median & 0.203364 \\
Maximum & 3.578526 \\
Minimum & -3.554012 \\
Std. Dev. & 1.437495 \\
Skewness & -0.223999 \\
Kurtosis & 2.802591 \\
Jarque-Bera & 0.709029 \\
Probability & 0.701514
\end{tabular}

FUENTE: INE y elaboración propia. 
TABLA 3

CORRELOGRAMA DE LOS RESIDUOS

Date: 12/20/21 Time: 19:41

Sample: 2001Q1 2019Q4

Q-statistic probabilities adjusted for 2 ARMA terms

\begin{tabular}{|c|c|c|c|c|c|c|}
\hline Autocorrelation & Partial Correlation & & $A C$ & PAC & Q-Stat & Prob* \\
\hline $\mathbf{I}$ & & 1 & -0.158 & $8-0.158$ & 1.8488 & \\
\hline I & I & 2 & 0.093 & $\begin{array}{ll}30.069\end{array}$ & 2.4938 & \\
\hline I & I & 3 & 0.012 & 20.037 & 2.5039 & 90.114 \\
\hline 1 & I & 4 & -0.109 & $9-0.112$ & 3.4287 & 0.180 \\
\hline I & I & 5 & 0.147 & $\begin{array}{ll}7 & 0.115\end{array}$ & 5.1248 & 30.163 \\
\hline , & I & 6 & 0.120 & 0.186 & 6.2811 & 0.179 \\
\hline 1 & & 7 & -0.149 & $9-0.138$ & 8.0737 & 0.152 \\
\hline I & I & 8 & 0.067 & $7-0.018$ & 8.4477 & 0.207 \\
\hline 1 & 1 & 9 & -0.248 & $8-0.200$ & 13.579 & 0.059 \\
\hline 1 & 1 & 10 & 0.049 & $9-0.004$ & 13.784 & 0.088 \\
\hline 1 & I & 11 & 0.113 & $\begin{array}{ll}30.110\end{array}$ & 14.886 & 0.094 \\
\hline I & I & 12 & -0.010 & 0.048 & 14.894 & 0.136 \\
\hline I & I & 13 & 0.086 & 0.072 & 15.561 & 0.158 \\
\hline I & I & 14 & 0.156 & 0.250 & 17.778 & 0.123 \\
\hline I & I & 15 & 0.029 & $\begin{array}{ll}9 & 0.183\end{array}$ & 17.858 & 0.163 \\
\hline I & 1 & 16 & 0.006 & $6-0.122$ & 17.861 & 0.213 \\
\hline 1 & 1 & 17 & 0.089 & 90.036 & 18.615 & 0.232 \\
\hline I & 1 & 18 & -0.088 & B -0.109 & 19.380 & 0.249 \\
\hline I & I & 19 & 0.086 & $6-0.026$ & 20.113 & 30.268 \\
\hline Iו & 1 & 20 & -0.150 & -0.196 & 22.407 & 0.214 \\
\hline 1 & 1 & 21 & -0.144 & $4-0.210$ & 24.560 & 0.176 \\
\hline 1 & I & 22 & 0.007 & $7 \quad 0.011$ & 24.565 & 0.219 \\
\hline I & 1 & 23 & -0.032 & 0.148 & 24.674 & 0.262 \\
\hline 1 & 1 & 24 & 0.011 & $\begin{array}{ll}1 & 0.104\end{array}$ & 24.687 & 0.312 \\
\hline 1 & 1 & 25 & 0.016 & $6-0.055$ & 24.715 & 50.365 \\
\hline 1 & 1 & 26 & -0.180 & -0.096 & 28.439 & 0.242 \\
\hline 1 & 1 & 27 & 0.160 & 0.105 & 31.464 & +0.174 \\
\hline 1 & 1 & 28 & 0.036 & $6 \quad 0.015$ & 31.619 & 0.206 \\
\hline I & 1 & 29 & 0.068 & $8-0.130$ & 32.190 & 0.225 \\
\hline 1 & 1 & 30 & 0.074 & $4-0.074$ & 32.874 & 0.240 \\
\hline 1 & 1 & 31 & -0.182 & $2-0.091$ & 37.171 & 0.142 \\
\hline 1 & 1 & 32 & -0.027 & $\begin{array}{ll}7 & 0.011\end{array}$ & 37.268 & 30.169 \\
\hline
\end{tabular}

*Probabilities may not be valid for this equation specification.

FUENTE: INE y elaboración propia. 